Elsevier required licence: () 2019. This manuscript version is made available under the CC-BY-NC-ND 4.0 license $\mathrm{http}: / /$ creativecommons.org/licenses/by-nc-nd/4.0/

The definitive publisher version is available online at $h$ ttps://doi.org/10.1016/j.jsv.2019.01.024 


\title{
Indirect bridge modal parameters identification with one stationary and one moving sensors and Stochastic Subspace Identification
}

\author{
Jiantao $\mathrm{Li}^{\mathrm{a}}$, Xinqun Zhu*a ${ }^{*}$, Siu-seong $\mathrm{Law}^{\mathrm{b}}$ and Bijan Samalic \\ ${ }^{a}$ School of Civil and Environmental Engineering, University of Technology Sydney, Broadway, NSW 2007, \\ Australia. ${ }^{1}$ Email: jacky.li@westernsydney.edu.au \\ *Corresponding author: xinqun.zhu@uts.edu.au \\ ${ }^{b}$ School of Civil Engineering, Chongqing University.Email: siu-seong.law@connect.polyu.hk \\ ${ }^{c}$ School of Computing, Engineering and Mathematics, Western Sydney University, Penrith, NSW 2751, \\ Australia.E-mail: b.samali@westernsydney.edu.au
}

\begin{abstract}
A new indirect strategy is proposed to estimate the bridge modal parameters from the dynamic responses of two vehicles using stochastic subspace identification technique. The effect of ambient excitation, such as ongoing traffic, is simulated as white-noise excitation at the bridge supports. The state-space model of the vehicle-bridge interaction system is derived for a single-degree-of-freedom quarter-car model and the bridge deck modeled as a simply-supported Euler-Bernoulli beam. Bridge modal frequencies can be estimated accurately from the vehicle responses. Two instrumented vehicles are required to estimate the bridge mode shapes, with one serving as a fixed reference sensor and the other as a moving sensor. The measured accelerations from the vehicles are divided into segments and each pair of signal segments forms a state-space identification problem. Local mode shape value from each signal segment can be estimated using the reference-based SSI method. A rescaling on the local mode shape values is applied to construct the global mode shapes. Effects of the bridge surface roughness, measurement noise and vehicle properties on the mode shape identification are also numerically studied. A vehicle-bridge interaction model in the laboratory serves for the experimental validation of the proposed strategy. Both numerical and experimental results show that the proposed method can estimate the bridge modal parameters with acceptable accuracy.
\end{abstract}

Keywords: indirect approach, modal parameter identification, stochastic subspace identification, vehiclebridge interaction, wireless monitoring system 


\section{Introduction}

Vibration-based structural health monitoring (SHM) has been studied widely in the last few decades [1]. The identification of modal parameters is important in most SHM approaches. Conventional identification approaches collect dynamic responses of the bridge directly. This poses a number of practical problems, such as the need of bridge closure, accidental damage to installed equipment, the high initial and recurring cost of the sensory system, etc.

Yang et al. [2] pioneered an indirect approach to estimate the bridge modal frequencies by analysing the acceleration response of an instrumented vehicle during its passage over the deck. The sensors were installed on the axle of the vehicle. The vehicle response mainly contains three types of frequencies, i.e., the driving frequency, vehicle modal frequency and the bridge modal frequency. The vehicle modal frequency usually appears as a dominant peak in the spectrum of the vehicle response, and this may mask the peaks of the bridge modal frequencies. This indirect approach without instrumentation on the bridge deck has the merits of more portable, convenient and cost-effective than the direct approach with an instrumented bridge. Recently, several hybrid methods have been proposed [3,4]. Chen et al. [5] presented a classification framework for indirect bridge SHM. Generalized pattern search algorithm was successfully applied to identify the bridge stiffness and its fundamental modal frequency from the vehicle responses [6]. A modified stochastic subspace identification technique was also used to extract the bridge modal frequencies from the response of a moving instrumented vehicle [7]. Lederman et al. [8] proposed a new supervised classification-based method to monitor rail infrastructures with vibration data collected from an operating passenger train. Four different features, i.e. temporal-frequency, spatial-frequency, spatial-amplitude, and signal-energy, were extracted from the vibration signals and examined. The signal-energy was found best for detecting physical changes of the track from both the simulated and the operational data.

Not many researchers have obtained the mode shapes of the bridge indirectly compared to the bridge modal frequencies [9]. Short-time frequency domain decomposition (FDD) was adopted to estimate the bridge mode shapes from the dynamic responses of a passing vehicle [10]. The quality of the estimated mode shape depends on the singular value at a selected frequency obtained by the peak-picking method. Some prior knowledge of the modal frequencies is required. Yang et al. [11] conducted a theoretical study for constructing the mode shape of a bridge using Hilbert transform. Kong et al. [12] used a test vehicle consisting of a tractor and two following trailers for their studies. Fast Fourier transformation and short-time Fourier transformation were applied to the residual response (difference) from two trailers to extract the frequencies and mode shape squares of the bridge deck, respectively. The latter was, however, found not satisfactory due to the limitation of the short-time Fourier transform. The above studies have not considered the effect of the tractor and/or ongoing traffic conditions. Marulanda et al. [13] used a mobile sensor and one fixed sensor on a simply supported beam to collect acceleration data. The modal frequencies of the beam could be easily obtained from 
measurement of the fixed sensor, and data from both sensors were used to identify the mode shapes. However, the effect of the properties of moving sensor on the identification was not studied. Malekjafarian and Obrien [14] presented a method for estimating the bridge mode shape from the response of a passing truck-trailer with an actuator using Hilbert-Huang transform. A rescaling process similar to the normalization of mode shape values in experimental modal analysis, was applied to the response amplitudes of two following axles to construct the mode shape. A few damage detection methods using the drive-by mode shape estimation were also developed. All the above studies on drive-by mode shape estimation are theoretical with little considerations on practical problems with field application. Zhang et al. [15] proposed a damage index based on the mode shape squares with laboratory verification. The damage index was used for structural damage detection with laser measurements from the moving vehicle [16]. Oshima et al. [17] compared the change of the mode shape due to the damage using the modal assurance criterion.

Stochastic subspace identification (SSI) is widely used for operational modal analysis to get the structural dynamic parameters from the bridge responses only [18]. The Reference-based Covariance-Driven SSI (RefSSI) was developed [19] for obtaining the global structural mode shapes. Measurements from a large structure may be divided into multiple setups with overlapping reference sensors. Stabilization diagrams were used to separate the true system poles from the spurious numerical poles.

This paper proposes a reference-based stochastic subspace-based method to obtain the bridge mode shapes from acceleration responses of one stationary and one moving instrumented vehicles. This simulates close-toreal scenarios with practical application of the drive-by technique. The ambient excitation, such as the ongoing traffic, is considered as white noise excitations at bridge supports. The stationary vehicle is at a fixed location serving as the reference sensor while the other one moves over the deck serving as a moving sensor. Measurements from the two vehicles are divided into segments corresponding to the same number of physical segments of the deck. The local mode shape values for each bridge segment is obtained from the corresponding measured data segment. It is then rescaled to construct the global mode shape vectors of the bridge deck. The effectiveness and performance of the proposed strategy are investigated numerically and experimentally with a laboratory structure. The bridge mode shapes can be identified efficiently with acceptable accuracy from the vehicle responses.

The rest of this paper is organised as follows. The modelling of the vehicle-bridge interaction system is presented followed by the derivation of the state space formulation of the Vehicle-Bridge Interaction (VBI) system. The Ref-SSI for the identification of bridge mode shapes from multiple measurement setups and the rescaling procedure is then presented. Numerical case studies are then conducted to demonstrate the effectiveness and performance of the proposed strategy. The effects of different vehicle speeds and physical 
parameters of vehicle on the identified results are also studied. Lastly, a vehicle-bridge model in the laboratory with wireless monitoring system is used for the experimental verification.

\section{The vehicle-bridge Interaction model in state space}

The vehicle is modelled as a single-degree-of-freedom (SDOF) quarter-car model and the bridge deck is simulated as a simply-supported Euler-Bernoulli beam.

\subsection{Equation of motion of the vehicle}

Considering the VBI system as shown in Figure 1, the vehicle is assumed to move along the simply-supported bridge deck at a constant velocity $v$. The equation of motion for the vehicle can be written as

$$
m_{v} \ddot{d}_{v}(t)+c_{v} \dot{d}_{v}(t)+k_{v} d_{v}(t)=\left\{c_{v}\left[\dot{d}_{b}(x, t)+v r^{\prime}(x)\right]+k_{v}\left[d_{b}(x, t)+r(x)\right]\right\}_{x=v t}
$$

where $m_{v}, k_{v}, c_{v}$ are the mass, stiffness and damping of the vehicle respectively, $d_{v}(t)$ is the vertical displacement of the vehicle, $d_{b}(x, t)$ is the vertical displacement of the bridge at the contact point $x$ and time $t$, and $r(x)$ is the road surface roughness with $r^{\prime}(x)=d r(x) / d x$.

\subsection{Equation of motion for the bridge deck}

According to the Euler-Bernoulli beam theory, the governing equation for flexural vibrations of the bridge deck under a moving vehicle can be written as:

$$
E I \frac{\partial^{4} d_{b}(x, t)}{\partial x^{4}}+\rho \frac{\partial^{2} d_{b}(x, t)}{\partial t^{2}}+\mu \frac{\partial d_{b}(x, t)}{\partial t}=-F(t) \delta(x-v t)
$$

where $E$ is Young's modulus of material; $I$ is the moment of inertia of deck cross-section; $E I$ is the flexural rigidity of deck; $\rho$ is the mass of material per unit length; $\mu$ is the viscous damping parameter; $F(t)$ is the interaction force between the bridge and vehicle with $F(t)=m_{v} g+m_{v} \ddot{d}_{v}(t)$, where $g$ is the acceleration of gravity, and $\delta$ is the Dirac function.

The displacement response of the deck can be expressed with modal superposition [20] as

$$
d_{b}(x, t)=\sum_{i=1}^{n} \phi_{i}(x) \eta_{i}(t)
$$

where $n$ is the number of modes considered. $\phi_{i}(x)=\sqrt{\frac{2}{\rho L}} \sin \frac{i \pi x}{L} \in R^{n \times 1}$ is the $i t h$ vibration mode shape, and $\eta_{i}(t)$ is the ith modal coordinate. $L$ is the length of the deck. 
Substituting Eq. (3) into Eq. (2) and applying the orthogonality conditions of vibration modes, Eq. (2) becomes:

$$
\ddot{\eta}_{i}(t)+2 \zeta_{i} \omega_{i} \dot{\eta}_{i}(t)+\omega_{i}^{2} \eta_{i}(t)=-F(t) \phi_{i}(v t)
$$

where $2 \zeta_{i} \omega_{i}=\mu / \rho$ and $\omega_{i}^{2}=\frac{E I}{\rho}\left(\frac{i \pi}{L}\right)^{4}$.

A bridge deck is subjected, in general, to multiple support excitations resulting from the on-going traffic, ground motion and wind excitation on deck, etc. This excitation is modeled in this paper as white noise time series applied at the right and left bridge supports denoted as $\ddot{d}_{r}(t)$ and $\ddot{d}_{l}(t)$, respectively [21]. The equation of motion for the $i$ th vibration mode of the deck subject to a moving vehicle and support excitations can then be written as

$$
\ddot{\eta}_{i}(t)+2 \zeta_{i} \omega_{i} \dot{\eta}_{i}(t)+\omega_{i}^{2} \eta_{i}(t)=-F(t) \phi_{i}(v t)+P_{i}(t)
$$

where $P_{i}(t)=-\ddot{d}_{l}(t) \int_{0}^{L} \rho\left(1-\frac{x}{L}\right) \phi_{i}(x) d x-\ddot{d}_{r}(t) \int_{0}^{L} \rho \frac{x}{L} \phi_{i}(x) d x$.

Combining Eqs. (1) and (5), the equation of motion of the vehicle-bridge interaction system can be obtained in matrix form as

$$
M \ddot{d}+\mathbf{C d}+K \mathbf{d}=\mathbf{F}
$$

in which

$$
\begin{aligned}
& \mathbf{M}=\left[\begin{array}{ccccc}
1 & 0 & \cdots & 0 & m_{v} \phi_{1}(x) \\
0 & 1 & \cdots & 0 & m_{v} \phi_{2}(x) \\
\vdots & \vdots & \ddots & 0 & \vdots \\
0 & 0 & \cdots & 1 & m_{v} \phi_{n}(x) \\
0 & 0 & 0 & 0 & m_{v}
\end{array}\right], \mathbf{C}=\left[\begin{array}{ccccc}
2 \zeta_{1} \omega_{1} & 0 & \cdots & 0 & 0 \\
0 & 2 \zeta_{2} \omega_{2} & \cdots & 0 & 0 \\
\vdots & \vdots & \ddots & 0 & \vdots \\
0 & 0 & \cdots & 2 \zeta_{n} \omega_{n} & 0 \\
-c_{v} \phi_{1}(x) & -c_{v} \phi_{2}(x) & \cdots & -c_{v} \phi_{n}(x) & c_{v}
\end{array}\right], \\
& \mathbf{K}=\left[\begin{array}{ccccc}
\omega_{1}^{2} & 0 & \cdots & 0 & 0 \\
0 & \omega_{2}^{2} & \cdots & 0 & 0 \\
\vdots & \vdots & \ddots & 0 & \vdots \\
0 & 0 & \cdots & \omega_{n}^{2} & 0 \\
-c_{v} \dot{\phi}_{1}(x)-k_{v} \phi_{1}(x) & -c_{v} \dot{\phi}_{2}(x)-k_{v} \phi_{2}(x) & \cdots & -c_{v} \dot{\phi}_{n}(x)-k_{v} \phi_{n}(x) & k_{v}
\end{array}\right] \\
& \mathbf{d}=\left[\begin{array}{c}
\eta_{1}(t) \\
\eta_{2}(t) \\
\vdots \\
\eta_{n}(t) \\
d_{v}(t)
\end{array}\right], \mathbf{F}=\left[\begin{array}{c}
-\phi_{1} m_{v} \mathrm{~g}+P_{1}(t) \\
-\phi_{2} m_{v} \mathrm{~g}+P_{2}(t) \\
\vdots \\
-\phi_{n} m_{v} \mathrm{~g}+P_{n}(t) \\
k_{v} r(x)+c_{v} v r^{\prime}(x)
\end{array}\right]
\end{aligned}
$$


The dynamic responses of the vehicle can be calculated as the 'measured' data by time-stepping integration from Eq. (6) using the explicit Newmark-Beta method [22]. They are then used to estimate the bridge modal parameters in this study.

\subsection{Formulation of VBI system in state space}

The vehicle-bridge interaction model in state space is introduced in this section [23]. When written in state space, Eq. (5) becomes

$$
\left\{\begin{array}{l}
\dot{\boldsymbol{\eta}}(t) \\
\ddot{\boldsymbol{\eta}}(t)
\end{array}\right\}=\left[\begin{array}{cc}
\mathbf{0} & \mathbf{I}_{n} \\
-\omega_{i}^{2} \mathbf{I}_{n} & -2 \zeta_{i} \omega_{i} \mathbf{I}_{n}
\end{array}\right]\left\{\begin{array}{l}
\mathbf{\eta}(t) \\
\dot{\boldsymbol{\eta}}(t)
\end{array}\right\}-\left[\begin{array}{c}
\mathbf{0} \\
m_{v} \mathbf{I}_{n}
\end{array}\right]\left[\begin{array}{ll}
\mathbf{0} & \mathbf{\Phi}(v t)
\end{array}\right]\left\{\begin{array}{l}
\dot{d}_{v}(t) \\
\ddot{d}_{v}(t)
\end{array}\right\}+\left[\begin{array}{c}
\mathbf{0} \\
m_{v} \mathbf{I}_{n}
\end{array}\right] g \boldsymbol{\Phi}(v t)+\left[\begin{array}{c}
\mathbf{0} \\
\mathbf{P}(t)
\end{array}\right]
$$

where $\boldsymbol{\eta}(\mathrm{t})=\left[\begin{array}{lllll}\eta_{1}(t) & \cdots & \eta_{i}(t) & \cdots & \eta_{n}(t)\end{array}\right] \in R^{n \times 1}, \boldsymbol{\Phi}(v t)=\left[\begin{array}{lllll}\phi_{1}(v t) & \cdots & \phi_{i}(v t) & \cdots & \phi_{n}(v t)\end{array}\right] \in$ $R^{n \times 1}, \mathbf{P}(\mathrm{t})=\left[\begin{array}{lllll}P_{1}(t) & \cdots & P_{i}(t) & \cdots & P_{n}(t)\end{array}\right] \in R^{n \times 1}$, and $\mathbf{I}_{n}$ is a unit matrix of size $n$.

Eq. (7) can then be written as

$$
\dot{\mathbf{X}}(t)=\mathbf{A}_{\mathbf{c}} \mathbf{X}(t)-\mathbf{B}_{\mathbf{c}} \mathbf{L}(t) \dot{\mathbf{Z}}(t)+\mathbf{B}_{\mathbf{c}} g \boldsymbol{\Phi}(v t)+\mathbf{P}_{\mathbf{c}}(t)
$$

where

$$
\begin{aligned}
& \mathbf{X}(t)=\left\{\begin{array}{l}
\mathbf{\eta}(t) \\
\dot{\boldsymbol{\eta}}(t)
\end{array}\right\} \in R^{2 n \times 1}, \mathbf{Z}(t)=\left\{\begin{array}{c}
d_{v}(t) \\
\dot{d}_{v}(t)
\end{array}\right\} \in R^{2 \times 1}, \mathbf{A}_{\mathbf{c}}=\left[\begin{array}{cc}
\mathbf{0} & \mathbf{I}_{n} \\
-\omega_{i}^{2} \mathbf{I}_{n} & -2 \zeta_{i} \omega_{i} \mathbf{I}_{n}
\end{array}\right] \in R^{2 n \times 2 n}
\end{aligned}
$$

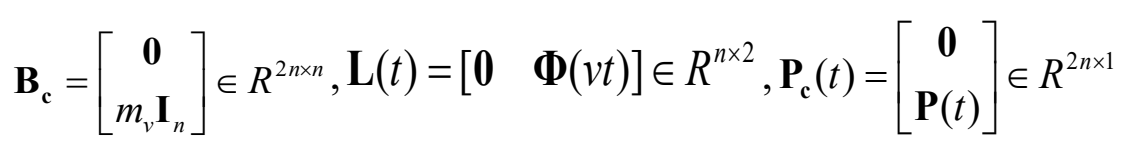

With the incorporation of an exponential matrix for transformation [17], Eq. (8) can be rewritten in a discretised recursive form as

$$
\mathbf{X}_{k+1}=\mathbf{A} \mathbf{X}_{k}-\mathbf{B L}_{k} \mathbf{Z}_{k}+\mathbf{B} g \mathbf{\Phi}_{k}+\mathbf{P}_{k}
$$

where $\mathbf{A}=\exp \left(\mathbf{A}_{\mathbf{c}} \cdot \Delta t\right), \mathbf{B L}_{k}=\mathbf{A}_{\mathbf{c}}^{-1}(\mathbf{A}-\mathbf{I}) \mathbf{B}_{\mathbf{c}} \mathbf{L}(t), \mathbf{P}_{k}=\mathbf{A}_{\mathbf{c}}^{-1}(\mathbf{A}-\mathbf{I}) \mathbf{P}_{\mathbf{c}}(t)$ and $\Delta t$ is the time step interval.

The second term on the right-hand-side (RHS) of Eq. (9) is the system input related to dynamic response $\mathbf{Z}_{k}$ of the moving vehicle. The third term is the input related to the weight of the vehicle and the fourth term is the ambient excitation. A light vehicle is assumed in this study, and the excitation due to the vehicle is much smaller compared with the on-going traffic excitation. The ambient excitation $\mathbf{P}_{k}$, which may consist of the on-going traffic excitation, will be dominating. The last three terms on the RHS in Eq. (9) can then be approximated by a stochastic process $\mathbf{v}_{k}$. 
Substitute Eq. (3), Eq. (1) can be re-written in state space as

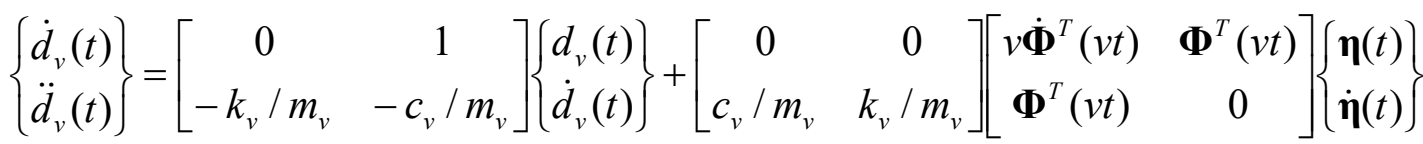

$$
\begin{aligned}
& +\left[\begin{array}{cc}
0 & 0 \\
k_{v} / m_{v} & c_{v} v / m_{v}
\end{array}\right]\left\{\begin{array}{c}
r(v t) \\
r^{\prime}(v t)
\end{array}\right\}
\end{aligned}
$$

or

$$
\dot{\mathbf{Z}}(t)=\mathbf{D}_{\mathbf{c}} \mathbf{Z}(t)+\mathbf{E}_{\mathbf{c}} \boldsymbol{\Phi}_{\mathbf{c}}(t) \mathbf{X}(t)+\mathbf{F}_{\mathbf{c}} \mathbf{R}(t)
$$

with

$$
\begin{aligned}
& \mathbf{Z}(t)=\left\{\begin{array}{c}
d_{v}(t) \\
\dot{d}_{v}(t)
\end{array}\right\}, \quad \mathbf{D}_{\mathbf{c}}=\left[\begin{array}{cc}
0 & 1 \\
-k_{v} / m_{v} & -c_{v} / m_{v}
\end{array}\right] \in R^{2 \times 2}, \mathbf{E}_{\mathbf{c}}=\left[\begin{array}{cc}
0 & 0 \\
c_{v} / m_{v} & k_{v} / m_{v}
\end{array}\right] \in R^{2 \times 2} \\
& \boldsymbol{\Phi}_{\mathbf{c}}(t)=\left[\begin{array}{cc}
v \dot{\boldsymbol{\Phi}}^{T}(v t) & \boldsymbol{\Phi}^{T}(v t) \\
\boldsymbol{\Phi}^{T}(v t) & 0
\end{array}\right] \in R^{2 \times 2 n}, \mathbf{F}_{\mathbf{c}}=\left[\begin{array}{cc}
0 & 0 \\
k_{v} / m_{v} & c_{v} v / m_{v}
\end{array}\right] \in R^{2 \times 2}, \mathbf{R}(t)=\left\{\begin{array}{l}
r(v t) \\
r^{\prime}(v t)
\end{array}\right\} \in R^{2 \times 1}
\end{aligned}
$$

Eq. (11) may be discretised as

$$
\mathbf{Z}_{k+1}=\mathbf{D Z} \mathbf{Z}_{k}+\mathbf{E} \boldsymbol{\Phi}_{k} \mathbf{X}_{k}+\mathbf{F} \mathbf{R}_{k}
$$

with $\mathbf{D}=\exp \left(\mathbf{D}_{\mathbf{c}} \cdot \Delta t\right), \mathbf{E} \boldsymbol{\Phi}_{k}=\mathbf{D}_{c}^{-1}(\mathbf{D}-\mathbf{I}) \mathbf{E}_{\boldsymbol{c}} \boldsymbol{\Phi}_{c}(t)$ and $\mathbf{F}=\mathbf{D}_{\mathbf{c}}^{-1}(\mathbf{D}-\mathbf{I}) \mathbf{F}_{\mathbf{c}}$.

Let $\mathbf{Y}_{k}=\mathbf{Z}_{k+1}-\mathbf{D Z} Z_{k}$ and $\mathbf{H}_{\boldsymbol{k}}=\mathbf{E} \boldsymbol{\Phi}_{k}$, and the road surface roughness related term, $\mathbf{F} \mathbf{R}_{k}$, is represented by a random stochastic process $\mathbf{w}_{k}$. The equation of motion for the VBI system in state space can be rewritten as

$$
\begin{gathered}
\mathbf{X}_{k+1}=\mathbf{A} \mathbf{X}_{k}+\mathbf{v}_{k} \\
\mathbf{Y}_{k}=\mathbf{H}_{k} \mathbf{X}_{k}+\mathbf{w}_{k}
\end{gathered}
$$

The bridge modal frequencies can be obtained from the vehicle responses in Eq. (13) using the SSI method.

\section{Subspace identification of the bridge mode shape from multiple measurement setups}

The application of the Ref-SSI method [19] requires dynamic responses at different locations of the structure to provide sufficient spatial information in the identification of bridge mode shapes. Two instrumented vehicles are used to measure the dynamic responses of the deck mimicking the presence of a large group of sensors along the deck. One vehicle will be located at a pre-selected location on the deck during measurement acting as the reference sensor and the other vehicle moves over the deck with a constant velocity. The bridge 
length is divided into $N$ physical segments. Measured signals from these two vehicles are also divided into $N$ corresponding segments. The measured signal from each segment of the deck may be assumed to come from different measurement setups representing the local dynamic behaviour of the deck at that segment. Two signal segments from the two vehicles form one pair of data record, and there will be $N$ pairs of records. All the observed output can be put together as

$$
\mathbf{Y}=\{\underbrace{\left(\begin{array}{c}
\mathbf{Y}^{(1, \text { ref })} \\
\mathbf{Y}^{(1, \text { mov })}
\end{array}\right)}_{\text {Record 1 }} \underbrace{\left(\begin{array}{c}
\mathbf{Y}^{(2, \text { ref })} \\
\mathbf{Y}^{(2, \text { mov })}
\end{array}\right)}_{\text {Re cord 2 }} \cdots \underbrace{\left(\begin{array}{c}
\mathbf{Y}^{(j, \text { ref })} \\
\mathbf{Y}^{(j, \text { mov })}
\end{array}\right)}_{\text {Re cord j }} \underbrace{\left(\begin{array}{c}
\mathbf{Y}^{(N, \text { ref })} \\
\mathbf{Y}^{(N, \text { mov })}
\end{array}\right)}_{\text {Re cord N }}\}
$$

where, $\mathbf{Y}^{(j, r e f)}$ and $\mathbf{Y}^{(j, m o v)}$ are the observed output vectors from the reference and the moving sensors on the jth segment of the deck, respectively.

Excitation within the duration of each record is assumed stationary within the short period of measurement. The state space formulation of the system including the deck, the reference and moving vehicles for the $j t h$ record of data $(j=1, \ldots, N)$ may be written as

$$
\left\{\begin{aligned}
\mathbf{X}_{k+1}^{(j)} & =\overline{\mathbf{A}} \mathbf{X}_{k}^{(j)}+\mathbf{V}_{k}^{(j)} \\
\mathbf{Y}_{k}^{(j, r e f)} & =\mathbf{H}^{(j, r e f)} \mathbf{X}_{k}^{(j)} \\
\mathbf{Y}_{k}^{(j, m o v)} & =\mathbf{H}^{(j, m o v)} \mathbf{X}_{k}^{(j)}
\end{aligned}\right.
$$

where $\mathbf{X}_{k}$ is the state vector at time instant $k, \mathbf{H}^{(j, r e f)}$ is the observation matrix from the reference sensor, $\mathbf{H}^{(j, m o v)}$ is the observation matrix from the moving sensor, $\overline{\mathbf{A}}$ is the state transition matrix, $\mathbf{V}_{k}$ is the unknown stationary noise vector, and $\mathbf{Y}_{k}^{j}=\left(\begin{array}{c}\mathbf{Y}_{k}^{(j, r e f)} \\ \mathbf{Y}_{k}^{(j, m o v)}\end{array}\right)$ is the vector of observed output. Due to the stationary assumption, the observation matrix in Eq. (15) becomes time-invariant within each record segment.

The Ref-SSI is then applied to the system in Eq. (15) in the following steps:

1) Determine the output covariance matrices between all outputs and references by

$$
\mathbf{R}_{i}^{r e f}=\mathbf{E}\left[\mathbf{Y}_{k+i} \mathbf{Y}_{k}^{(r e f)^{T}}\right]
$$

2) Form a block Toeplitz matrix using the covariance matrices

$$
\mathbf{T}_{i}^{r e f}=\left[\begin{array}{cccc}
\mathbf{R}_{i}^{r e f} & \mathbf{R}_{i-1}^{r e f} & \cdots & \mathbf{R}_{1}^{r e f} \\
\mathbf{R}_{i+1}^{r e f} & \mathbf{R}_{i}^{r e f} & \cdots & \mathbf{R}_{2}^{r e f} \\
\cdots & \cdots & \cdots & \cdots \\
\mathbf{R}_{2 i-1}^{r e f} & \mathbf{R}_{2 i-2}^{r e f} & \cdots & \mathbf{R}_{i}^{r e f}
\end{array}\right]
$$


The block matrix can then be decomposed as

$$
\mathbf{T}_{i}^{r e f}=\left(\begin{array}{c}
\mathbf{H} \\
\mathbf{H} \overline{\mathbf{A}} \\
\cdots \\
\mathbf{H}^{i-1}
\end{array}\right)\left(\begin{array}{lllll}
\overline{\mathbf{A}}^{i-1} \mathbf{G} & \overline{\mathbf{A}}^{i-2} \mathbf{G} & \cdots & \overline{\mathbf{A}} \mathbf{G} & \mathbf{G}
\end{array}\right)=\boldsymbol{O}_{i} C_{i}^{r e f}
$$

where $\boldsymbol{C}_{i}^{r e f}=\left(\begin{array}{lllll}\overline{\mathbf{A}}^{\boldsymbol{i}-\mathbf{1}} \mathbf{G} & \overline{\mathbf{A}}^{\boldsymbol{i}-\mathbf{2}} \mathbf{G} & \cdots & \overline{\mathbf{A}} \mathbf{G} & \mathbf{G}\end{array}\right)$ is the controllability matrix, $\mathbf{G}=\mathbf{E}\left[\mathbf{X}_{k+1} \mathbf{Y}_{k}^{(r e f)^{\boldsymbol{T}}}\right]$, and $\boldsymbol{O}_{i}=\left(\begin{array}{llll}\mathbf{H} & \mathbf{H} \overline{\mathbf{A}} & \cdots & \mathbf{H} \\ \overline{\mathbf{A}}^{\boldsymbol{i}-\mathbf{1}}\end{array}\right)^{T}$ is the observability matrix

3) Calculate the observability matrix $\boldsymbol{O}_{i}$ and controllability matrix $\boldsymbol{C}_{i}^{r e f}$ by applying the singular-value decomposition to the block Toeplitz matrix as

$$
\mathbf{T}_{i}^{r e f}=\boldsymbol{U S} \boldsymbol{V}^{T}=\left(\begin{array}{ll}
\boldsymbol{U}_{1} & \boldsymbol{U}_{2}
\end{array}\right)\left(\begin{array}{cc}
\boldsymbol{S}_{1} & \mathbf{0} \\
\mathbf{0} & \mathbf{0}
\end{array}\right)\left(\begin{array}{l}
\boldsymbol{V}_{1}^{T} \\
\boldsymbol{V}_{2}^{T}
\end{array}\right)
$$

where $\boldsymbol{U}$ and $\boldsymbol{V}$ are orthonormal matrices and $\boldsymbol{S}$ is a diagonal matrix containing the singular values in descending order. The null singular values and corresponding singular vectors are omitted in Eq. (19). Matrix $\boldsymbol{S}_{1}$ is diagonal, containing non-zero singular values. Matric $\boldsymbol{U}_{1}, \boldsymbol{U}_{2} \boldsymbol{V}_{1}$ and $\boldsymbol{V}_{2}$ are portions of $\boldsymbol{U}$ and $\boldsymbol{V}$ related to $\boldsymbol{S}_{\mathbf{1}}$, respectively. The system order can be determined by inspecting the number of non-zero singular values. Stabilisation diagram is constructed to eliminate spurious mode due to noise. Inspection of Eqs. (18) and (19) gives

$$
\begin{aligned}
& \boldsymbol{O}_{i}=\boldsymbol{U}_{1} \boldsymbol{S}_{1}^{1 / 2} \\
& \boldsymbol{C}_{i}^{r e f}=\boldsymbol{S}_{1}^{1 / 2} \boldsymbol{V}_{1}^{T}
\end{aligned}
$$

Once $\boldsymbol{O}_{i}$ and $\boldsymbol{C}_{i}^{r e f}$ are obtained, the state matrix $\overline{\mathbf{A}}$ can then be retrieved to obtain the eigenvalues and eigenvectors $\left(\lambda_{j}, \varphi_{\lambda, j}\right)$ of the system [24].

The Reference-based SSI method is then applied to each data record $j(=1, \ldots, N)$ to obtain the local mode shape values at the mid-point of each deck segment. Since the reference sensor is the same for all records, the observation matrix $H^{(r e f)}$ is the same for all measurement records.

A progressive rescaling procedure is applied to estimate the global mode shape. It is similar to the normalization procedure in experimental modal analysis of a structure. After the local mode shape values of each segment are obtained, they are re-scaled with respect to that at the reference DOF to form the global mode shape. For example, when the bridge is divided into five segments as shown in Figure 2, the measurement data from the two vehicles is divided into five pairs of data records. For the first data set, the local mode shape value for the first bridge segment and reference location are $\boldsymbol{\varphi}_{1,1}$ and $\boldsymbol{\varphi}_{1, \text { ref }}$ respectively. If the reference sensor is located at the middle of the first segment, the local mode shape values of the first data set from the moving and reference sensors are measured at the same time, and therefore they can be treated as 
the global mode shape values with $\boldsymbol{\Phi}_{1} \approx \boldsymbol{\Phi}_{\text {ref }}$. For the second data set, the local mode shape value for the second and reference segments are obtained as $\boldsymbol{\varphi}_{2,2}$ and $\boldsymbol{\varphi}_{2, \text { ref }}$ respectively. To obtain the global mode shape of the second segment $\boldsymbol{\Phi}_{2}$, a rescaling factor is employed with $\boldsymbol{\Phi}_{2}=\frac{\boldsymbol{\Phi}_{\text {ref }}}{\boldsymbol{\varphi}_{2, \text { ref }}} \boldsymbol{\varphi}_{2,2}$. The calculated local mode shape value in each segment can be rescaled to the global ones as

$$
\boldsymbol{\Phi}_{j}=\frac{\boldsymbol{\Phi}_{r e f}}{\boldsymbol{\varphi}_{j, r e f}} \boldsymbol{\varphi}_{j, j}, \quad(j=2,3, \cdots N)
$$

where $\Phi_{j}$ is the global mode shape value for the $j$ th segment. The accuracy of this approach depends on the number of segments in the sub-divisionn of the recorded signal. Therefore, a suitable number of segments have to be chosen to balance the required spatial information for the analysis and the accuracy of the identified global mode shapes.

\section{Numerical simulations}

The numerical example by Yang and Chen [7] is adopted to validate the proposed method. Parameters of the undamped bridge are $L=30 \mathrm{~m}, \rho=10700 \mathrm{~kg} / \mathrm{m}, I=0.494 \mathrm{~m}^{4}, E=29.43 \mathrm{GPa}$. Damping of the bridge is neglected because it is insignificant to the forced vibration of the bridge under moving vehicle action [11]. The first three modal frequencies of the bridge are $2.03,8.12$ and $18.30 \mathrm{~Hz}$, respectively. The properties for the undamped vehicle are $m_{v}=100 \mathrm{~kg}, k_{v}=170 \mathrm{kN} / \mathrm{m}$. The modal frequency of the vehicle is $6.50 \mathrm{~Hz}$ and the moving speed is $2 \mathrm{~m} / \mathrm{s}$. The duration of data for the analysis is 15 seconds and the sampling rate is $1000 \mathrm{~Hz}$. The amplitude of ambient excitation at the supports is $0.02 \mathrm{~m} / \mathrm{s}^{2}$. These parameters are adopted for all studies in this paper unless otherwise stated.

\subsection{Modal frequency identification using one instrumented vehicle}

Previous study [25] has indicated that the vehicle response is sensitive to the bridge surface roughness. Classes A and B road surface roughness are therefore considered in this study. The damping properties of the vehicle and bridge are not considered in this section. The vehicles start moving $50 \mathrm{~m}$ before entering the bridge, and the road surface roughness is assumed the same as that of the bridge. The dynamic responses of the vehicle are given in Figure 3(a) and the corresponding spectra are shown in Figure 3(b). Only the vehicle frequency is noted dominating the spectra. The peak-picking method for FDD cannot get the bridge modal frequencies from the spectra. The stabilization diagram in the SSI analysis with Class A road roughness shown in Figure 4(a) features the first two bridge modal frequencies as well as the vehicle modal frequency. The third bridge modal frequency is, however, too small to be noted. When the vehicle moves on deck with Class B road 
roughness, the stabilization diagram in Figure 4(b) shows only the vehicle frequency, and the bridge frequencies are not present. Yang et al. [26] have proposed the idea of subtracting the measured acceleration responses of two following axles with the same properties travelling over a bridge. This serves to reduce the effect of road roughness. However, the formulation of this approach has not considered the interaction between the two following axles and that of the tractor.

\subsection{Identification of bridge mode shapes using two instrumented vehicles}

The acceleration responses from sensors on both vehicles are analysed. The reference vehicle is located at mid-span and quarter span to capture a larger modal amplitude for the identification of first and second mode shapes respectively. The segmentation scheme is such selected to ensure that the vibration amplitude at the midpoint of each segment is not zero. This configuration is adopted in the following studies unless otherwise stated.

\subsubsection{Two vehicles on a smooth road surface}

In the first case, the bridge deck is divided into 5 and 9 segments. The dynamic responses of the two vehicles and their spectra with the smooth deck are given in Figure 5. The two time histories are divided into 5 and 9 segments according to the bridge segmentation and the corresponding $\boldsymbol{\Phi}_{\text {ref }}$ are $\boldsymbol{\Phi}_{3, \text { ref }}, \boldsymbol{\Phi}_{5, \text { ref }}$, at mid-span respectively. For the identification of the second mode shape, the dynamic responses of the two vehicles are divided into 6 or 10 segments, and the corresponding $\boldsymbol{\Phi}_{\text {ref }}$ are $\boldsymbol{\Phi}_{2, \text { ref }}, \boldsymbol{\Phi}_{4, \text { ref }}$, at quarter-span respectively.

The Ref-SSI method is applied to each data segment to obtain the local mode shape value at each bridge segment. The identified first and second mode shapes after the rescaling process are given in Figure 6. Excellent agreement is found for the first mode shape with the MAC value equals 0.998 and 0.999 for the cases with 5 and 9 segments, respectively. For the second mode shape in Figure 6(b), the agreement between the theoretical and identified results is slightly poorer. It is, however, noted that the MAC value increases when a larger number of segments is chosen for both mode shape identification. It may be concluded that a suitable number of segments have to be selected to balance the need of spatial information and the accuracy of the identified mode shapes in the analysis.

\subsubsection{Two vehicles on a rough road surface}

The effects of Class A and Class B bridge surface roughness are studied using 9 and 10 segments for the $1^{\text {st }}$ and $2^{\text {nd }}$ modes, respectively. When the reference vehicle is at mid-span of the deck, the dynamic responses of two vehicles with Class A surface roughness are shown in Figure 7. The identified first mode shape is given in Figure 8(a). The MAC value decreases from 0.9999 to 0.9993 compared to that when the bridge is smooth. The identified second mode shape is given in Figure $8(\mathrm{~b})$ and it also associated with a slight decrease in the 
MAC value with Class A roughness. However, when a Class B bridge road surface roughness is considered, the MAC values for the identified first and second mode shapes are 0.7409 and 0.4843 , respectively indicating a set of poorly identified results.

\subsubsection{The effect of measurement noise}

The more practical case with contaminated measurements from the vehicle-bridge system is studied. The VBI system with Class A bridge surface roughness is studied using 9 and 10 segments for the $1^{\text {st }}$ and $2^{\text {nd }}$ modes, respectively. White noise is added to the calculated acceleration response of the vehicle to simulate the "polluted" measurements as

$$
a c c_{m}=a c c_{c a l}+E_{p} \bullet N_{o i s e} \bullet \sigma\left(a c c_{c a l}\right)
$$

where $a c c_{c a l}$ is the calculated acceleration response; $E_{p}$ is the noise level; $N_{\text {oise }}$ is a vector consisting of random values with zero mean and unit standard deviation; and $\sigma\left(a c c_{c a l}\right)$ is the standard deviation of the calculated acceleration response. Three noise levels, i.e. 5\%, 10\% and 15\% are considered and the corresponding signal-to-noise ratios are 26,20 and 16 , respectively.

The identified first and second mode shapes from using the proposed method are shown in Figures 9(a) and 9(b), respectively. The accuracy of the identified mode shapes is noted to decrease with an increase of the noise level. When the noise level is less than $10 \%$, the accuracy of the identified mode shapes is acceptable with the smallest MAC value equals to 0.99 . When the noise level reaches $15 \%$, the dynamic information of a few bridge segments is masked by the noise effect and the overall mode shapes cannot be identified. It may be concluded that the noise effect has to be minimized before the proposed method is applied for a better identification accuracy.

\subsection{The effect of moving speeds and vehicle parameters}

Five percent white noise is included in all numerical studies in this section and Class A road surface roughness is included.

\subsubsection{Effect of the vehicle speed}

Moving speeds of $2 \mathrm{~m} / \mathrm{s}, 4 \mathrm{~m} / \mathrm{s}$ and $6 \mathrm{~m} / \mathrm{s}$ are studied. The sampling frequency is $1000 \mathrm{~Hz}$ for $2 \mathrm{~m} / \mathrm{s}$ and $4 \mathrm{~m} / \mathrm{s}$, and $3000 \mathrm{~Hz}$ for $6 \mathrm{~m} / \mathrm{s}$ to ensure sufficient number of data in the sub-divisions. The identified first and second mode shape value for each segment are shown in Figures 10(a) and 10(b), respectively. When the moving speed increases to $4 \mathrm{~m} / \mathrm{s}$, the MAC values are 0.9786 and 0.9870 for the first two mode shapes respectively. When the speed is $6 \mathrm{~m} / \mathrm{s}$, the MAC values are 0.9526 and 0.9225 respectively for the first two mode shapes. All the MAC 
values are larger than 0.9 , and they are noted to drop with increasing speed. It may be concluded that a slower vehicle speed may be advantageous to the identification of mode shapes using the proposed indirect method.

\subsubsection{Effect of vehicle parameters}

Previous studies in this paper have shown the feasibility of the proposed method to obtain for the bridge modal properties from measured vehicle responses. The effects of the vehicle parameters on the identification are studied in this section. There are three parameters in the SDOF vehicle model, i.e. mass $m_{v}$, stiffness $k_{v}$ and damping $c_{v}$. Eq. (1) can be rewritten as

$$
m_{v} \ddot{d}_{v}(t)+c_{v} \dot{d}_{v}(t)+k_{v} d_{v}(t)=f_{b}(t)+f_{r}(t)
$$

where $f_{b}(t)=\left\{c_{v}\left[\dot{d}_{b}(x, t)\right]+k_{v}\left[d_{b}(x, t)\right]\right\}_{x=v t}$ and $f_{r}(t)=\left\{c_{v}\left[v r^{\prime}(x)\right]+k_{v}[r(x)]\right\}_{x=v t}$. The response spectrum of the vehicle can be obtained as

$$
D_{v}(w)=H_{v}(w) F_{b}(w)+H_{v}(w) F_{r}(w)
$$

where $H_{v}(w)=\frac{m_{v}}{1-w^{2}+2 j \xi_{v} w_{v} w}$ is the frequency response function of the vehicle. $\omega_{v}=\sqrt{k_{v} / m_{v}}$ and $\xi_{v}=\frac{c_{v}}{2 m_{v} w_{v}}$ are the vehicle frequency and damping ratio respectively. $F_{b}(w)$ is the input spectrum due to the bridge vibration and $F_{r}(w)$ is the spectrum due to the road surface roughness.

Figure 11 shows the amplitudes of the frequency response function with three different damping ratios 0.1 , 0.25 and 0.5 . The frequency ratio is defined as the ratio between the excitation frequency and the vehicle modal frequency. In order for the vehicle to effectively capture the bridge vibration components, it is preferable to have the dynamic magnification factor (DMF) not less than unity. Figure 11 shows that when the frequency ratio is less than 0.2 , the DMF is almost unity and the effects of damping can be neglected. When the frequency ratio ranges from 0.2 to 1.5 , a larger damping ratio would notably lead to a smaller dynamic magnification factor. When the frequency ratio is larger than 1.5, the magnification factors are all less than unity. The effect of damping ratio becomes negligible when the frequency ratio keeps increasing up to 2.0 . These results indicate that the selection of vehicle parameters should be considered carefully for a good identification accuracy.

Another study on the effects of vehicle parameters is also conducted. The location of reference vehicle is at mid-span and quarter-span respectively for the identification of the first and second mode shapes. Cases with 9 
and 10 segments are studied. Class A road surface roughness and 5\% noise are considered and the sampling rate is $1000 \mathrm{~Hz}$ in the simulation.

\section{a) Effect of the vehicle weight}

Vehicle weight of $100 \mathrm{~kg}, 200 \mathrm{~kg}$ and $500 \mathrm{~kg}$ are selected with $2.0 \mathrm{~m} / \mathrm{s}$ vehicle speed. The corresponding mass ratios between the vehicle and the bridge deck are $3.12 \times 10^{-4}, 6.23 \times 10^{-4}$ and $1.56 \times 10^{-3}$ respectively. The frequency ratios between the fundamental frequency of the deck and the vehicle frequency are $0.31,0.44$ and 0.69 respectively. They are 1.24, 1.75 and 2.77 respectively for the second bridge frequency. The identified results are presented in Figures 12(a) and 12(b). The identified first mode shape with 100kg weight vehicle is much closer to the true mode shape than those from $200 \mathrm{~kg}$ and $500 \mathrm{~kg}$ weight vehicles. The MAC values are $0.9982,0.9968$ and 0.9913 for $100 \mathrm{~kg}, 200 \mathrm{~kg}$ and $500 \mathrm{~kg}$ weight vehicles, respectively. The MAC value of the first mode shape is noted to decrease with increase in the vehicle weight. This is due to the proximity of the bridge and vehicle modal frequencies making the identification difficult. Results for the second mode shape are similar as shown in Figure 12(b) with the corresponding MAC values equal to 0.9950, 0.9908 and 0.9920, respectively. It is noted in Figure 13 that the dynamic magnification factor is reduced when the frequency ratio increases within the range of 0.2 to 1.5 . Eq. (9) also states that the effect of the vehicle weight on the total excitation term cannot be ignored when it is large. This may suggest that a lighter vehicle should be used with the proposed method.

\section{b) Effect of the vehicle stiffness}

The vehicle stiffness is chosen as $170 \mathrm{kN} / \mathrm{m}, 340 \mathrm{kN} / \mathrm{m}$ and $700 \mathrm{kN} / \mathrm{m}$ respectively. The vehicle mass, damping ratio and moving speed are $100 \mathrm{~kg}, 0.10$ and $2.0 \mathrm{~m} / \mathrm{s}$ respectively. They correspond to vehicle modal frequencies of $6.50 \mathrm{~Hz}, 9.19 \mathrm{~Hz}$ and $13.19 \mathrm{~Hz}$ respectively. The identified first and second mode shapes from using the proposed strategy are shown in Figure 13(a) and 13(b) respectively. The MAC values for the first mode shape are $0.9982,0.9964$ and 0.9988 respectively which match well with the true values. The MAC values for the second mode shape are $0.9950,0.9817$ and 0.9767 . The MAC value is reduced when the vehicle stiffness increases. This is because the vehicle with the high stiffness also captures the high frequency noise that includes the effect of road surface roughness. This effect has been shown as the second item in Eq. (27).

\section{c) Effect of the vehicle damping}

Three different damping ratios $0.10,0.25$ and 0.40 are studied. The vehicle mass and stiffness are $100 \mathrm{~kg}$ and $170 \mathrm{kN} / \mathrm{m}$, respectively. The vehicle is moving along the bridge at $2.0 \mathrm{~m} / \mathrm{s}$. The identified first and second bridge mode shapes are presented in Figures 14(a) and 14(b), respectively. The identified results in Figure 14(a) are very close to each other with a high MAC value relative to the theoretical ones. In this study, the 
frequency ratio is 0.31 , and the effect of the damping ratio is negligible as shown in Figure 11 . The identified second bridge mode shape is shown in Figure 14(b). The frequency ratio corresponding to the second bridge frequency is 1.24 with a magnification factor close to unity as shown in Figure 11. When the damping ratio is considered, the accuracy of the identified results decreases with larger damping as discussed earlier.

\section{Experimental verification}

\subsection{Experimental setup}

A vehicle-bridge interaction model was built in the laboratory as shown in Figure 15. The model consists of three rectangular steel beams as shown in Figure 15(a) and $100 \mathrm{~mm}$ wide and $15 \mathrm{~mm}$ deep steel beams. The main beam in the middle is continuous simply-supported over two spans with $3 \mathrm{~m}$ each. A leading beam and a trailing beam are sitting in front of and behind the main beam to allow for acceleration and deceleration of the vehicle and the length of these beams is $3 \mathrm{~m}$. Figure 15(b) shows the instrumentation of the bridge with wired sensors at the bottom surface of the beam for modal test. Vehicle model of different configurations with one and two-axle are fabricated for the study. The two-axle vehicle model has four steel wheels. It is used to simulate the unknown traffic on the bridge. One-axle vehicle is fabricated to simulate the SDOF model towed by the two-axle vehicle model. The mass of the one- and two-axle vehicles is 2 and $4 \mathrm{~kg}$, respectively. A BeanDevice AX-3D wireless sensor was installed on each vehicle to measure the vertical acceleration. An Ushaped aluminium section was glued along the centre of the upper surface of the beams as direction guide for the vehicles. The model vehicles were pulled along the guide with an electric motor. Figure 15(c) shows the vehicles on the beam. Laser sensors were installed to record the time instants when the vehicles arrive at and exit from the main beam. These time instants are used to calculate the moving speed of the vehicle.

Before the vehicle-bridge interaction test, modal test were conducted on the bridge and vehicle models with hammer impact excitation. The PCB352C34 accelerometers from PCB Piezotronics were used to measure the dynamic responses. Eight accelerometers were placed evenly on the bridge beam to collect data for experimental identification of the mode shape. National Instruments data acquisition system was used. The first modal frequency of the bridge was identified as $5.68 \mathrm{~Hz}$ and that of the single-axle vehicle is $29.37 \mathrm{~Hz}$. The corresponding mode shape was also identified from the modal test.

In the vehicle-bridge interaction test, a wireless sensory system was setup with devices manufactured by Beanair Gmbh. BeanDevice AX-3D wireless accelerometer was selected to measure the dynamic responses. Two wireless sensors were used with one installed on the single-axle vehicle as the moving sensor and one installed on the bridge beam (at $1.1 \mathrm{~m}$ to the right support) as the reference sensor, as shown in Figure 16. BeanGateway was used to control the wireless sensors.

\subsection{Experimental results}


Three different cases with varying weights of the two-axle vehicle are considered. No extra weight is added in Case 1. 2.5 and 5kg-weights are loaded on the two-axle vehicle, respectively in Cases 2 and 3. Notations $\mathrm{m} 1$, $\mathrm{m} 2$ and $\mathrm{m} 3$ are used to denote these three cases. The vehicles were pulled with three different speeds at approximately $0.10,0.14$ and $0.21 \mathrm{~m} / \mathrm{s}$, respectively which are denoted respectively by v1, v2 and v3 in the following discussions. Five sets of data from different test configurations were analyzed including the vehicle with three different weight moving with speed $v 2$ and the case $\mathrm{m} 2$ moving with two speeds.

Measurements from the moving and stationary sensors were analyzed with the proposed method to identify the first mode shape of the beam in each of the five tests. Each signal is divided into eight segments. The identified operational mode shape values are compared with those from modal tests using MAC values. Figure 17 presents the identified results for the three cases when the moving speed is v2. When no extra weight is added on the vehicle, the MAC value is about 0.98 , which is quite accurate relative to that experimental mode shape. When the weight of the two-axle vehicle increases, the MAC value of the identified results decreases. This may be due to stronger non-stationary interaction between the vehicle and the bridge models when under the heavier vehicle. This has been discussed with the third term on the RHS of Eq. (9) when the vehicle weight is large compared to the total mass of the bridge. Figure 18 shows the results for the weight case $\mathrm{m} 2$ with three different moving speeds. The MAC value decreases from about 0.98 to 0.94 when the speed increases from 0.10 to $0.21 \mathrm{~m} / \mathrm{s}$. This observation gives experimental evidences that a slower moving speed tends to provide more accurate identified results.

\subsection{Improve the accuracy of the experimental identification results by averaging}

For the weight case $\mathrm{m} 2$ with moving speed v2, two more tests were conducted by locating the reference sensor at $1.8 \mathrm{~m}$ and $2.6 \mathrm{~m}$ to the right support. The MAC value of the identified result are respectively 0.9630 and 0.9457. The latter location of the reference sensor is very close to the middle support, and the MAC value is small. The identified results are shown in Figure 19. However, when the three sets of identified mode shapes values are averaged as shown in the figure, the MAC value improves to 0.9731. This indicates that the accuracy of the identified results can be improved by averaging results obtained from different locations of the reference sensor.

\section{Conclusions}

A multiple-setup Reference-based SSI method is proposed to identify the bridge modal parameters using two instrumented vehicles. It is experimentally validated with a vehicle-bridge interaction model equipped with wireless monitoring system in the laboratory. One vehicle remains stationary during the whole procedure as the reference sensor, and the other vehicle measures the dynamic response while moving over the bridge simultaneously. Numerical and experimental results show that the proposed method is effective and robust to 
identify the bridge modal parameters from dynamic responses of two vehicles. When the bridge surface roughness is Class $\mathrm{A}$ and the measurement noise is less than $10 \%$, the bridge mode shapes can be estimated with good accuracy. When the bridge has a Class B surface roughness, the effect of the road roughness should be reduced before the proposed method can be used. However, the quality of the identified mode shape can be significantly improved by averaging results obtained from different locations of the reference sensor. The proposed method is also found to give more accurate results with a slow moving speed and a lighter vehicle.

\section{Acknowledgements}

This research is supported by research funding of the Australian Research Council Discover Project (DP160103197). The financial aid is gratefully acknowledged.

\section{References}

[1] Carden, EP and Fanning, P 2004, 'Vibration based condition monitoring: a review', Structural Health Monitoring, vol. 3, no. 4, pp. 355-77.

[2] Yang, YB, Lin, CW and Yau, JD 2004, 'Extracting bridge frequencies from the dynamic response of a passing vehicle', Journal of Sound and Vibration, vol. 272, no. 3, pp. 471-93.

[3] Malekjafarian, A, McGetrick, PJ and OBrien, EJ 2015, 'A review of indriect bridge monitoring using passing vehicles', Shock Vibration, 286139.

[4] Zhu, XQ and Law, SS 2015, 'Structural health monitoring based on vehicle-bridge interaction: accomplishments and challenges', Advances in Structural Engineering, vol.18, no. 12, pp.1999-2015.

[5] Chen, S, Cerda, F, Rizzo, P, Bielak, J, Garrett, JH and Kovačević, J 2014, 'Semi-supervised multiresolution classification using adaptive graph filtering with application to indirect bridge structural health monitoring', IEEE Transactions on Signal Processing, vol. 62, no. 11, pp. 2879-93.

[6] Li, WM, Jiang, ZH, Wang, TL and Zhu, HP 2014, 'Optimization method based on Generalized Pattern Search Algorithm to identify bridge parameters indirectly by a passing vehicle', Journal of Sound and Vibration, vol. 333, no. 2, pp. 364-80.

[7] Yang, YB and Chen, WF 2015, 'Extraction of bridge frequencies from a moving test vehicle by stochastic subspace identification', Journal of Bridge Engineering ASCE, vol. 21, no. 3, p. 04015053.

[8] Lederman, G, Chen, S, Garrett, J, Kovačević, J, Noh, HY and Bielak, J 2017, 'Track-monitoring from the dynamic response of an operational train', Mechanical Systems and Signal Processing, vol. 87, pp. 1-16.

[9] Yang, YB and Yang, JP 2017, 'Sate-of-the-art review on modal identification and damage detection of bridges by moving test vehicles', International Journal of Structural Stability and Dynamics, vol. 18, no. 2, 1850025.

[10] Malekjafarian, A and OBrien, EJ 2014, 'Identification of bridge mode shapes using Short Time Frequency Domain Decomposition of the responses measured in a passing vehicle', Engineering Structures, vol. 81, pp. 386-97.

[11] Yang, YB, Li, YC and Chang KC 2014, 'Constructing the mode shapes of a bridge from a passing vehicle: a theoretical study', Smart Structures and Systems, vol. 13, no. 5, pp. 797-819. 
[12] Kong, X, Cai, CS and Kong, B 2016, 'Numerically extracting bridge modal properties from dynamic responses of moving vehicles', Journal of Engineering Mechanics ASCE, vol. 142, no. 6, 04016025.

[13] Marulanda, J, Caicedo, JM and Thomson, P 2016, 'Modal identification using mobile sensors under ambient excitation', Journal of Computing in Civil Engineering ASCE, vol.31, no.2, 04016051.

[14] Malekjafarian, A and Obrien, EJ, 2017, 'On the use of a passing vehicle for the estimation of bridge mode shapes', Journal of Sound and Vibration, vol. 397, pp.77-91.

[15] Zhang, Y, Wang, LQ and Xiang, ZH, 2012, 'Damage detection by mode shape squares extracted from a passing vehicle', Journal of Sound and Vibration, vol. 331, no. 2, pp. 291- 307.

[16] Oshima, Yoshinobu, Kyosuke Yamamoto, and Kunitomo Sugiura. "Damage assessment of a bridge based on mode shapes estimated by responses of passing vehicles." Smart Structures and Systems, 13.5 (2014): $731-753$.

[17] Obrien, EJ and Malekkiafarian, A 2016, 'A mode shape- based damage detection approach using laser measurement from a vehicle crossing a simply supported bridge', Structural Control and Health Montiroing, vol. 23, no. 10, pp.1273-1286.

[18] Hong, AL, Ubertini, F and Betti, R 2012, 'New stochastic subspace approach for system identification and its application to long-span bridges', Journal of Engineering Mechanics ASCE, vol. 139, no. 6, pp. 724-36.

[19] Peeters, B and De Roeck, G 1999, 'Reference-based stochastic subspace identification for output-only modal analysis', Mechanical Systems and Signal Processing, vol. 13, no. 6, pp. 855-78.

[20] Clough, RW and Penzien, J 2003, Dynamics of Structures, Third Edition, Computers and Structures, Inc., Berkeley, CA USA.

[21] Zhang, YH, Li, QS, Lin, JH and Williams, FW 2009, 'Random vibration analysis of long-span structures subjected to spatially varying ground motions', Soil Dynamics and Earthquake Engineering, vol. 29, no.4, pp.620-629.

[22] Liu, K, Law, SS, Zhu, X and Xia, Y 2014, 'Explicit form of an implicit method for inverse force identification', Journal of Sound and Vibration, vol. 333, no. 3, pp. 730-44.

[23] Van Overschee, P and De Moor, B 1996, Subspace Identification for Linear Systems: Theory, Implementation, Applications. Kluwer Academic Publishers.

[24] Döhler, M, Andersen, P and Mevel, L 2011, 'Data merging for multi-setup operational modal analysis with data-driven SSI', in Structural Dynamics, vol. 3, Springer, pp. 443-52.

[25] Chang, KC, Wu, FB and Yang, YB 2010, 'Effect of road surface roughness on indirect approach for measuring bridge frequencies from a passing vehicle', Interaction and multiscale mechanics, vol. 3, no. 4, pp. 299-308.

[26] Yang, YB, Li, YC, and Chang, KC 2012. "Using two connected vehicles to measure the frequencies of bridges with rough surface: a theoretical study." Acta Mechanica, vol. 223, no. 8, 1851-1861. 


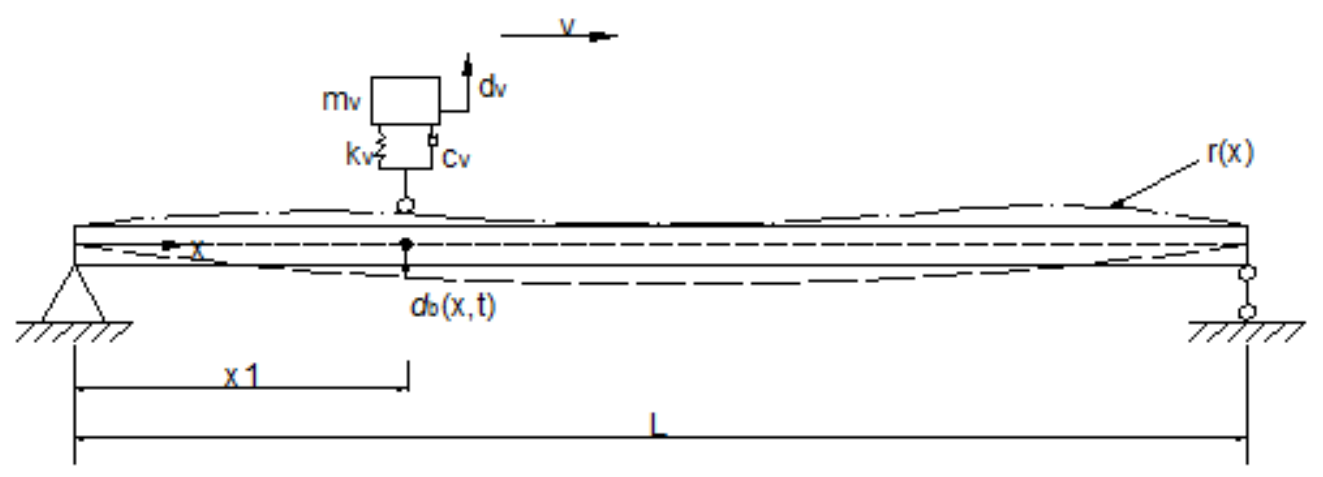

Figure 1 Vehicle-bridge system

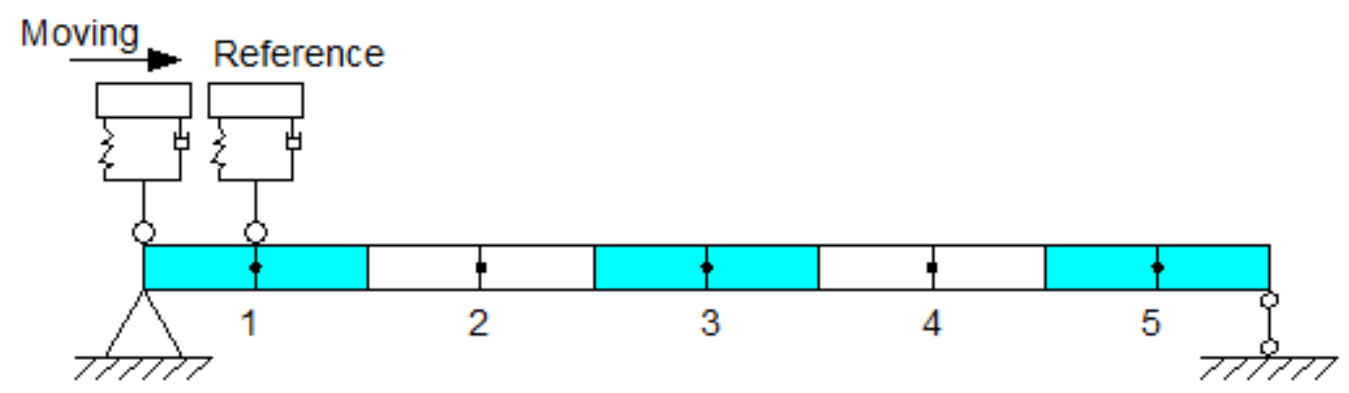

Figure 2 Measurement setup 

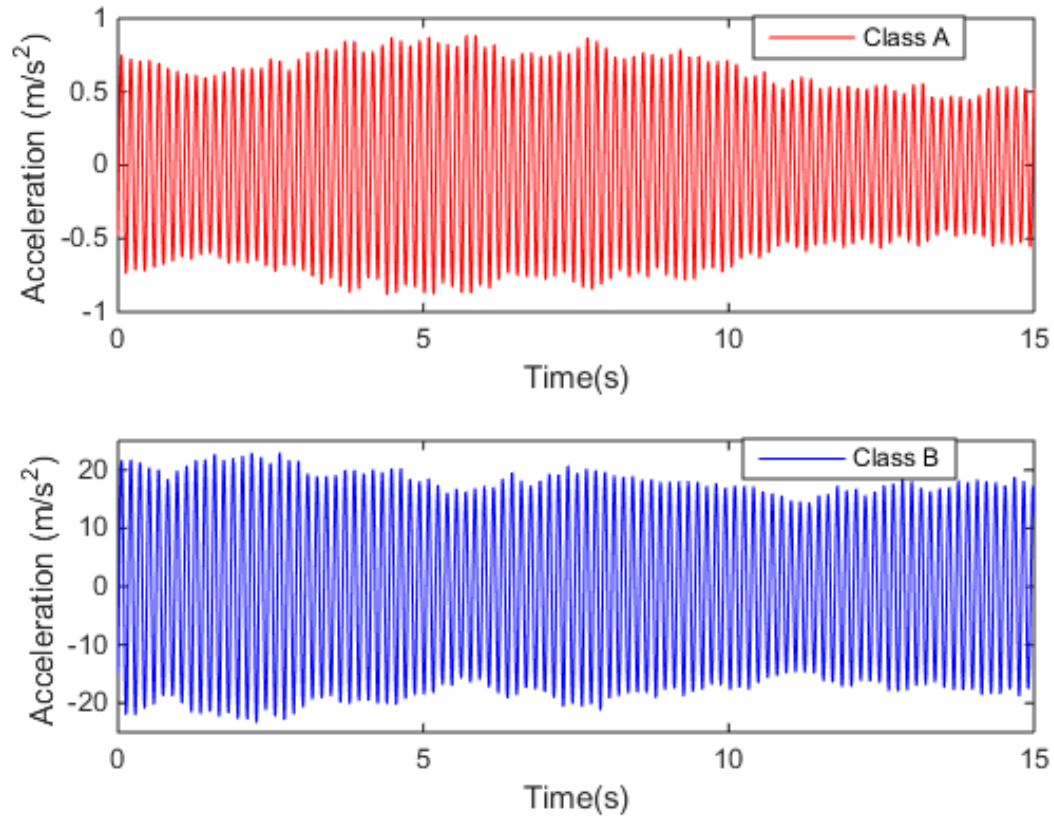

(a) Dynamic responses of the vehicles

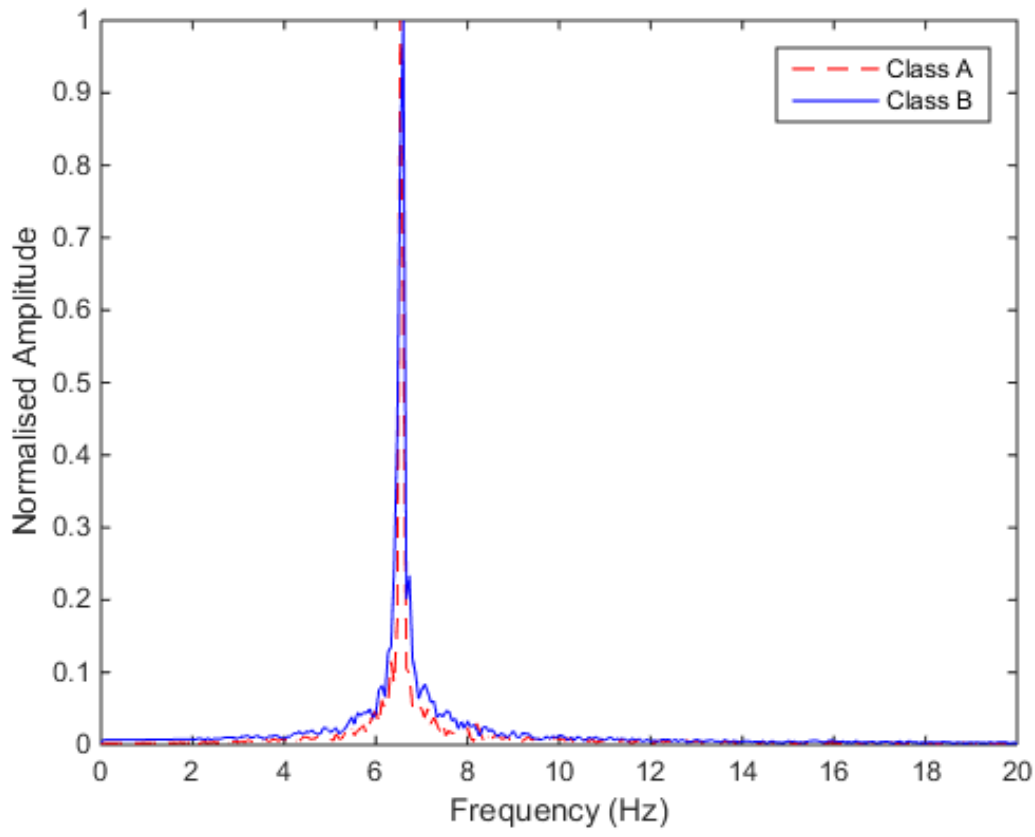

(b) Normalised spectrum of the vehicle responses

Figure 3 Dynamic responses and their spectrum of the vehicle moving on the bridge with Classes A and B surface roughness 


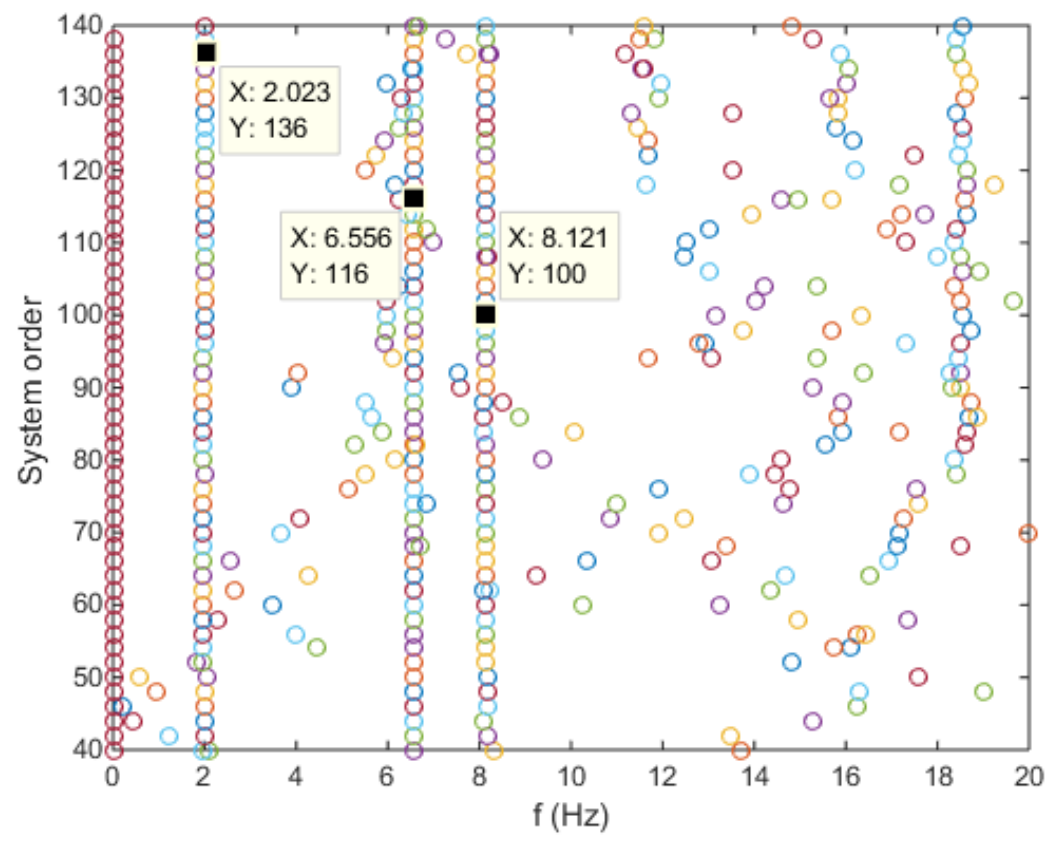

(a) Stabilization diagram with Class A surface roughness

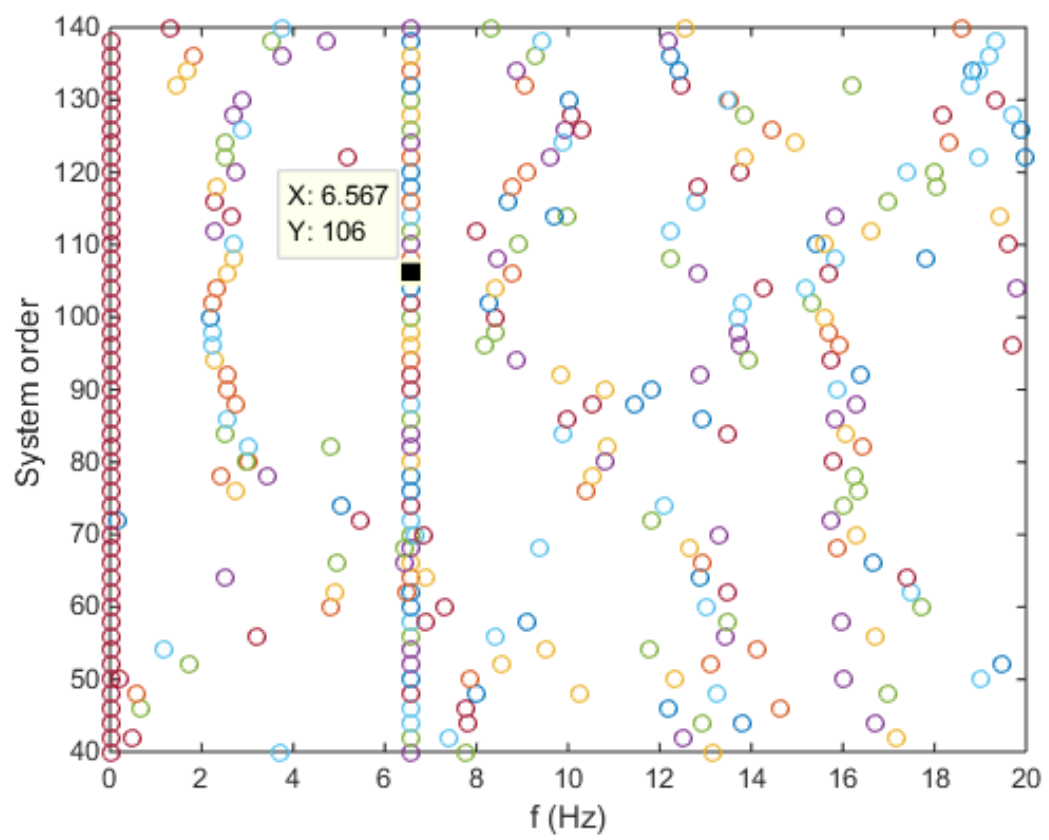

(b) Stabilization diagram with Class B surface roughness

Figure 4 Identified results of the bridge from the response of one vehicle 


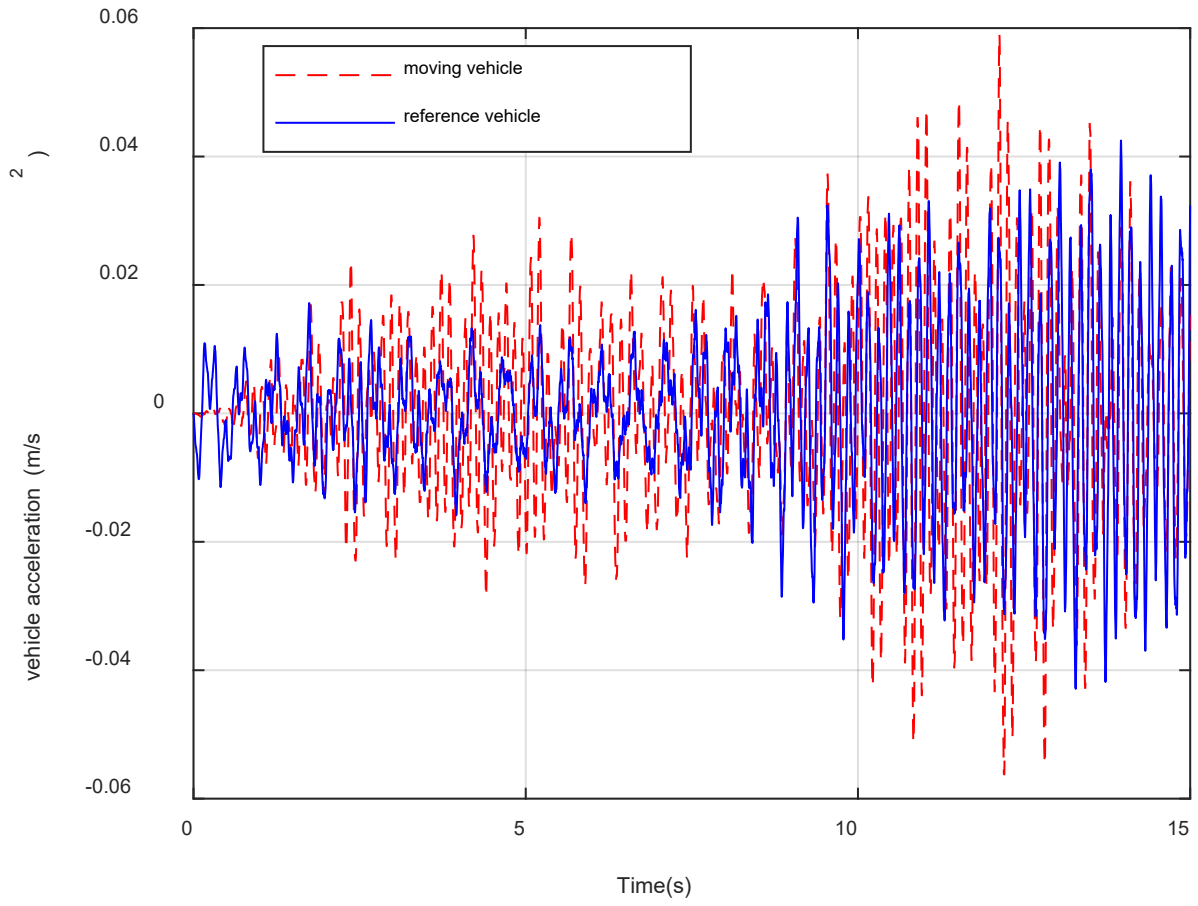

(a) Dynamic responses of two vehicles

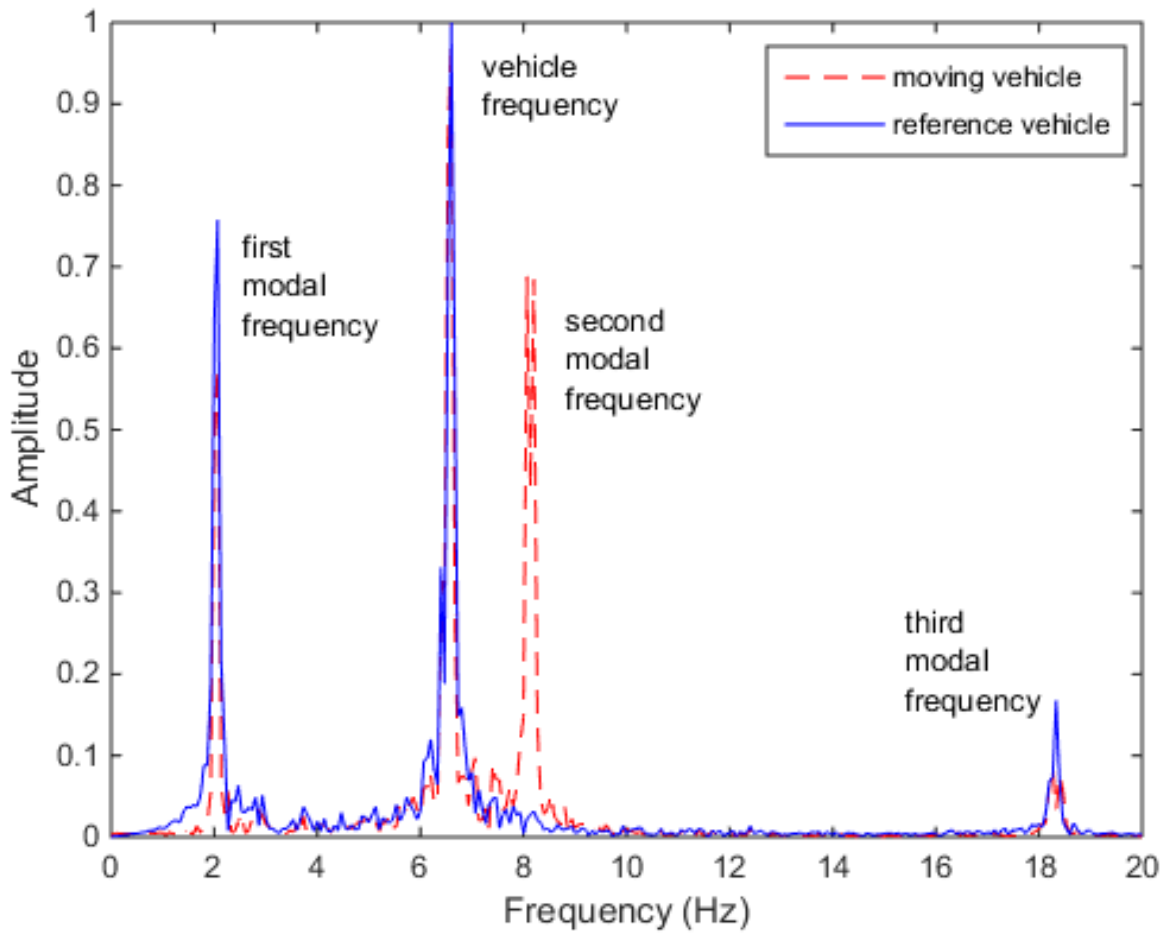

(b) Their spectra

Figure 5 Dynamic responses of the moving and reference vehicles and their spectra 


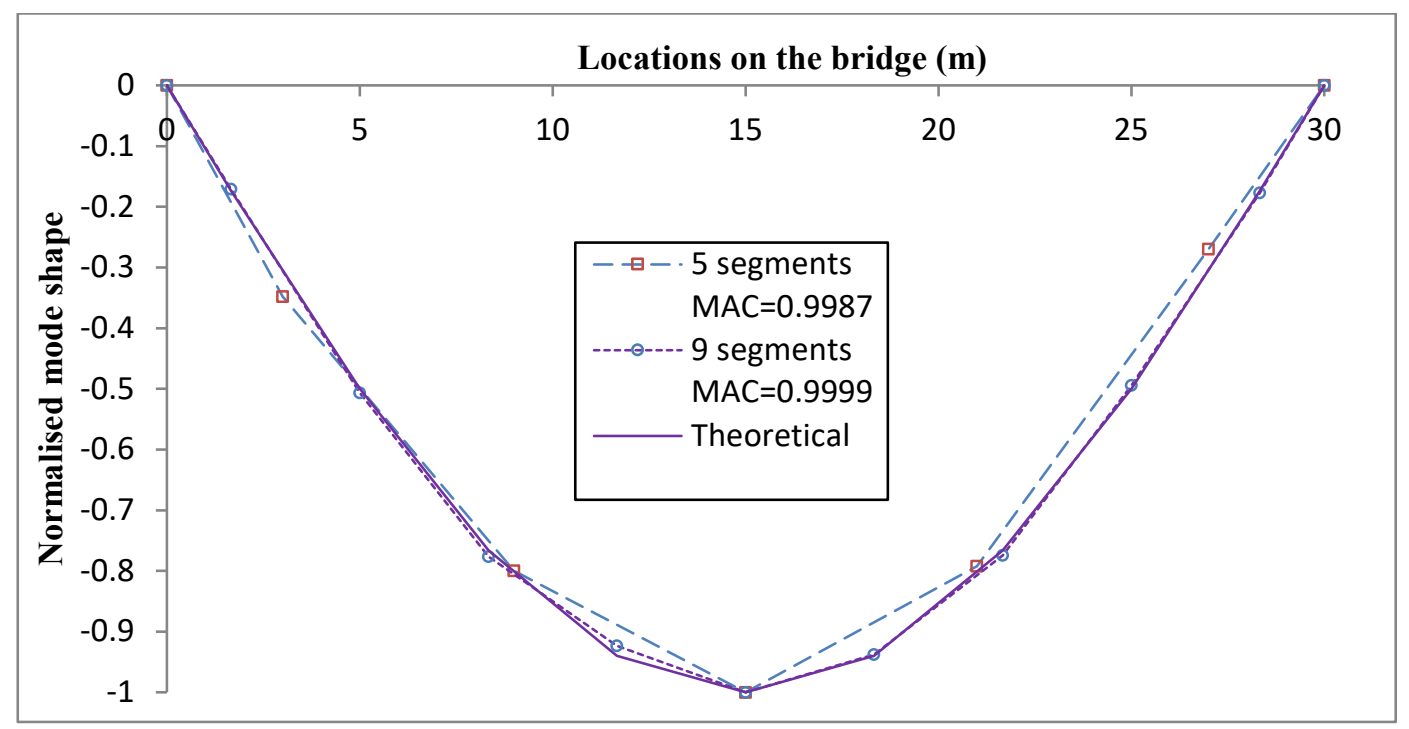

(a) Identified first mode shape with 5 and 9 segments

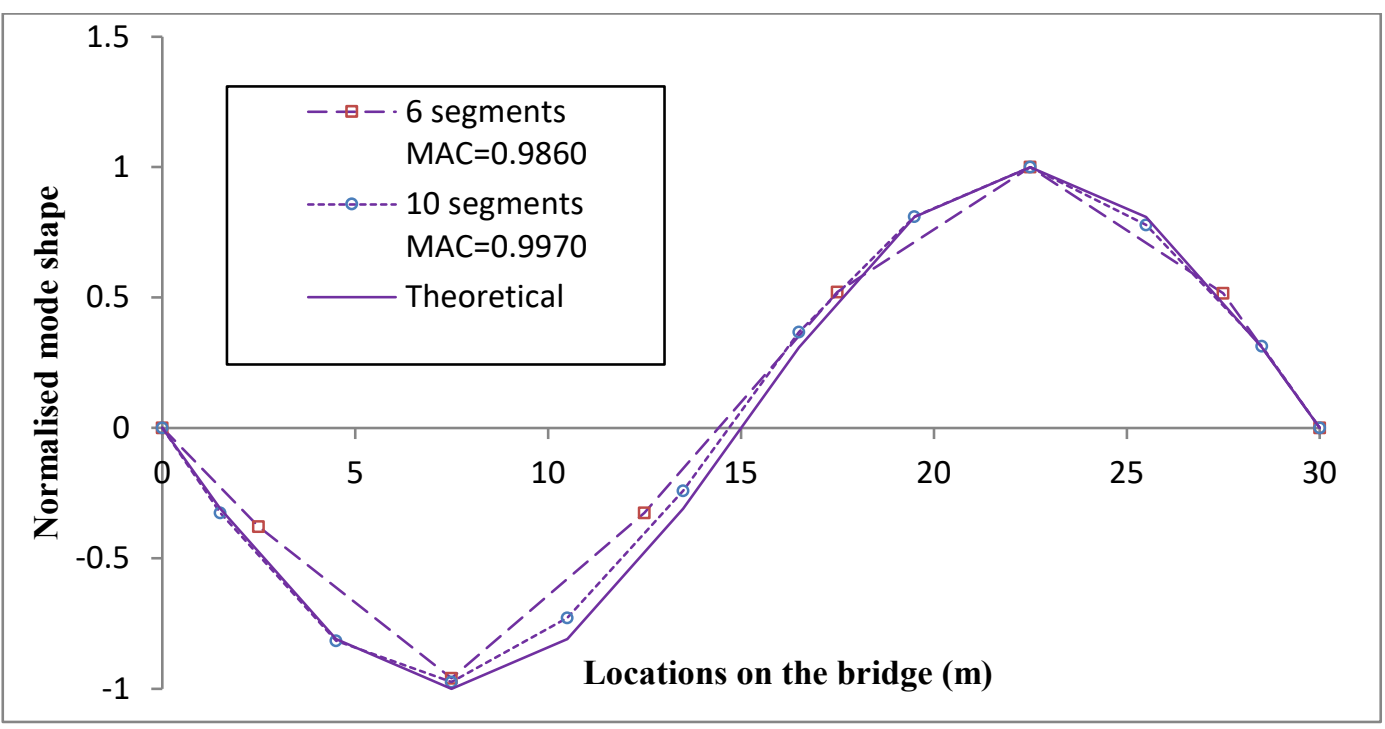

(b) Identified second mode shapes with 6 and 10 segments

Figure 6 Identified mode shapes with different segment numbers 


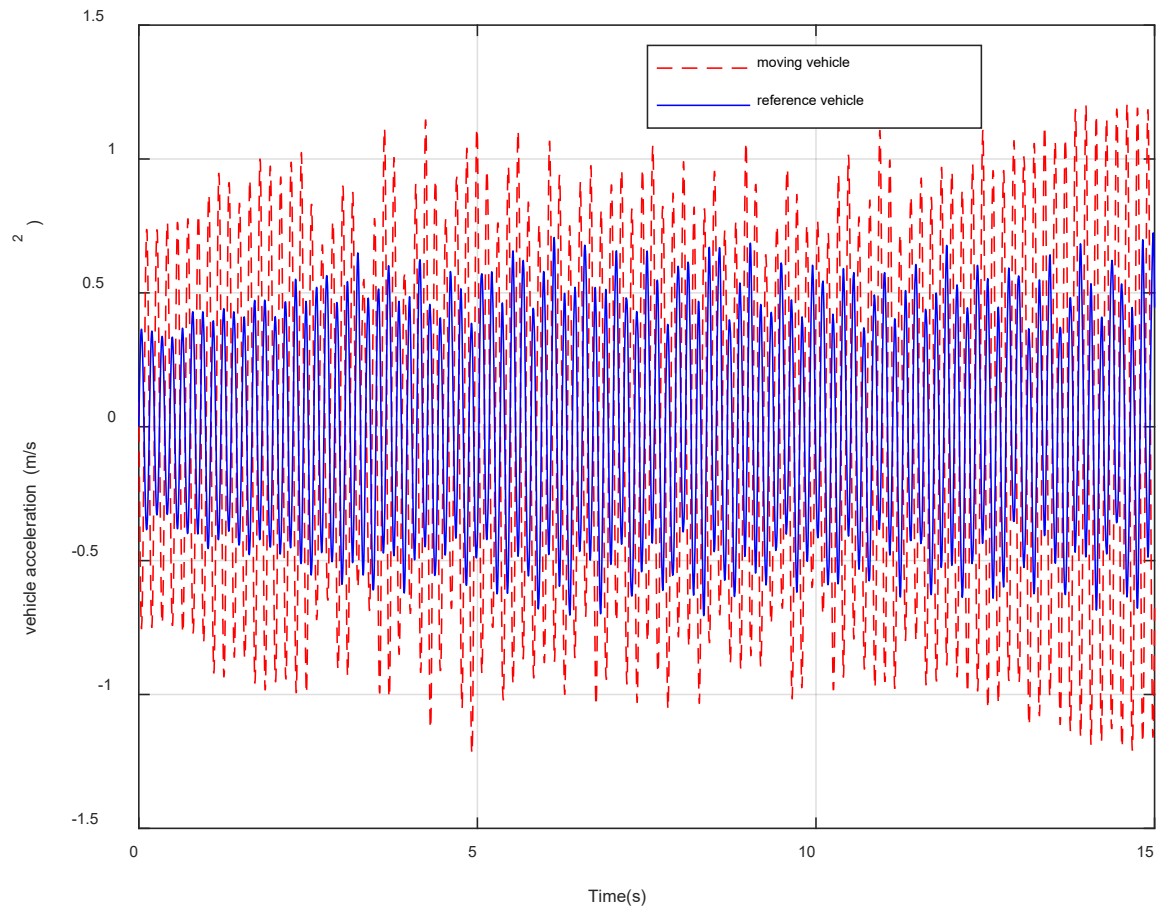

Figure 7 Dynamic responses of the vehicles when the surface roughness is Class A 


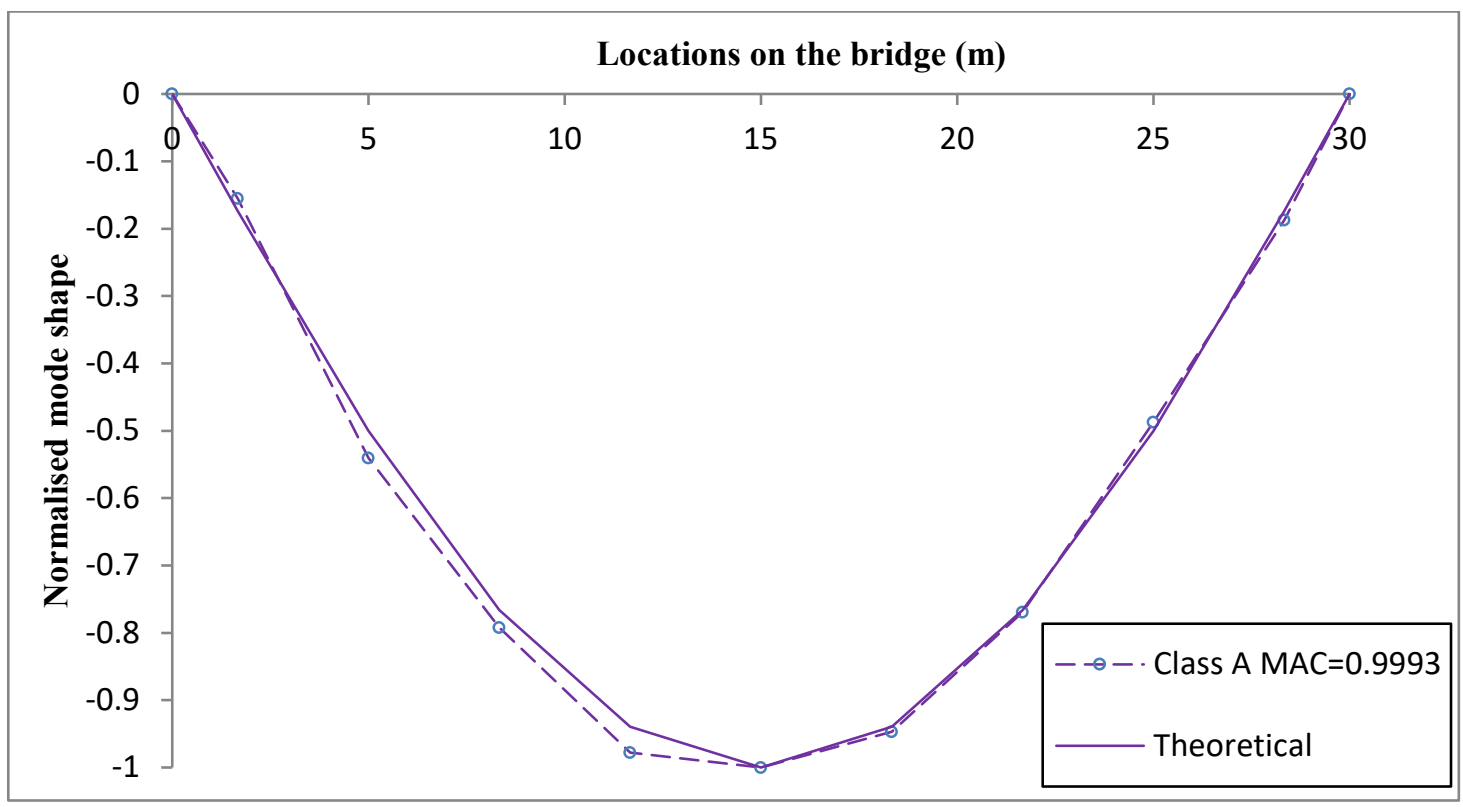

(a) Identified first mode shape

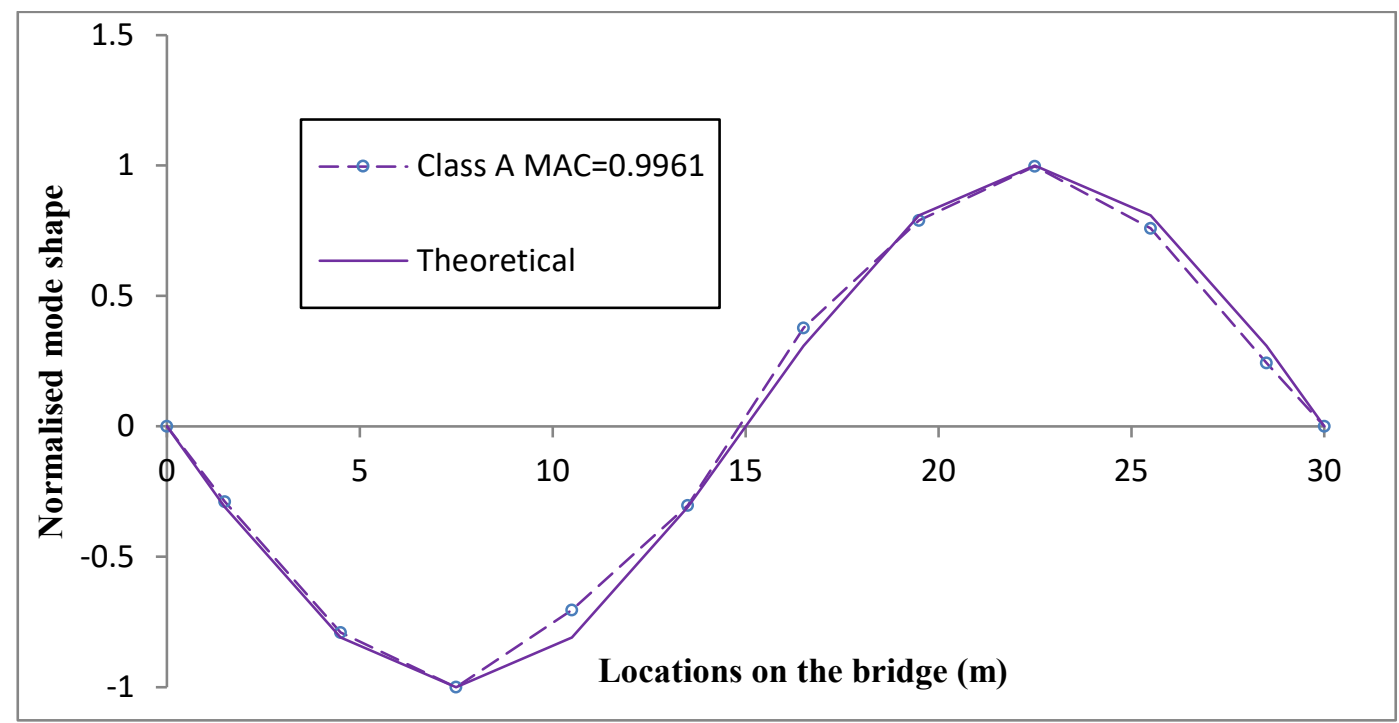

(b) Identified second mode shape

Figure 8 Identified mode shapes of the bridge with Class A roughness 


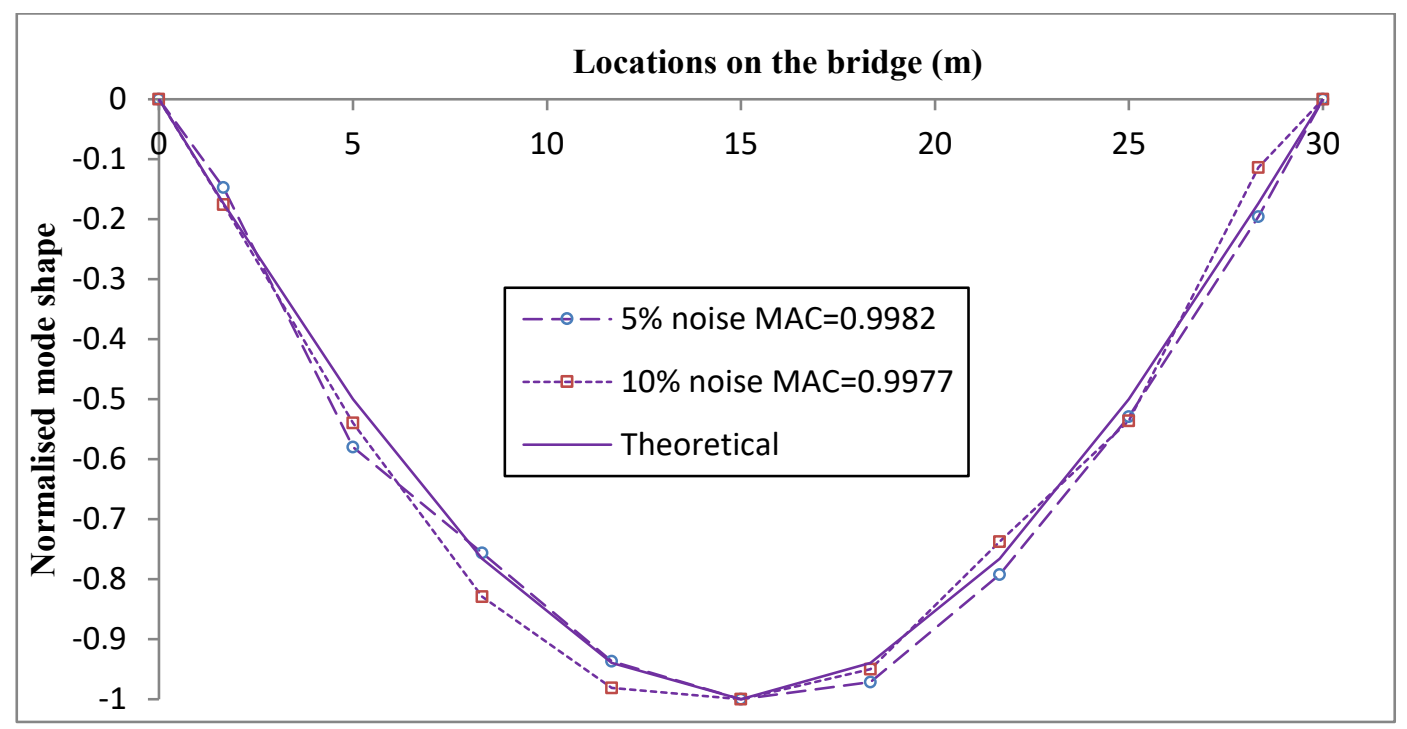

(a) Identified first mode shape

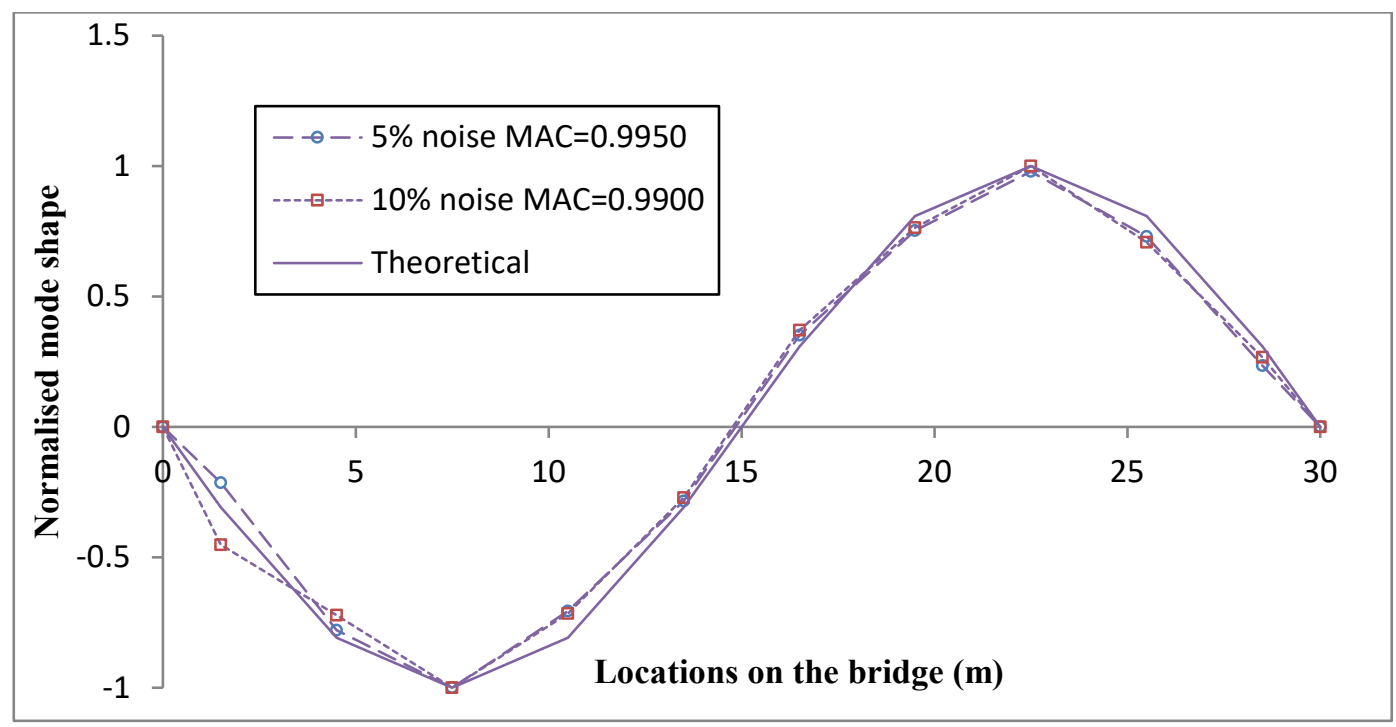

(b) Identified second mode shape

Figure 9 Effect of measurement noise on identified results 


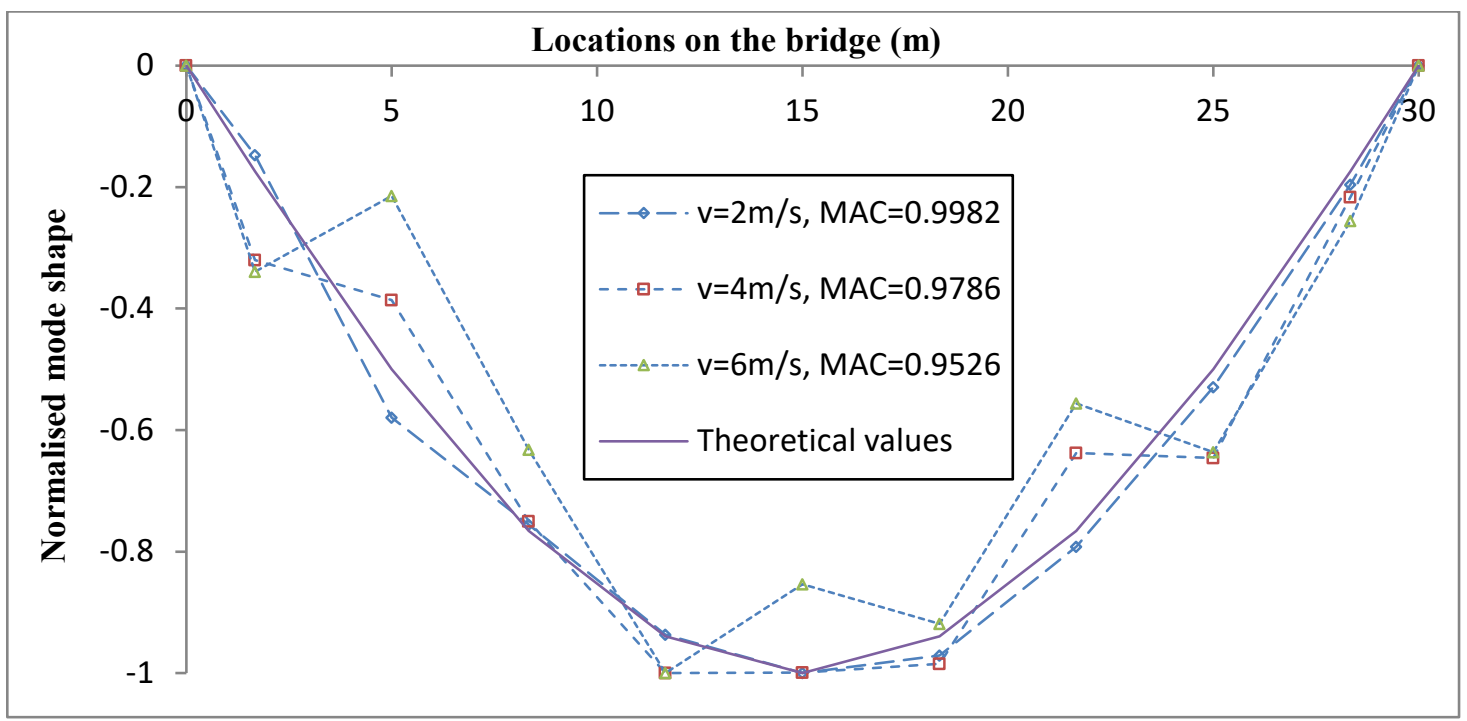

(a) Identified first mode shape

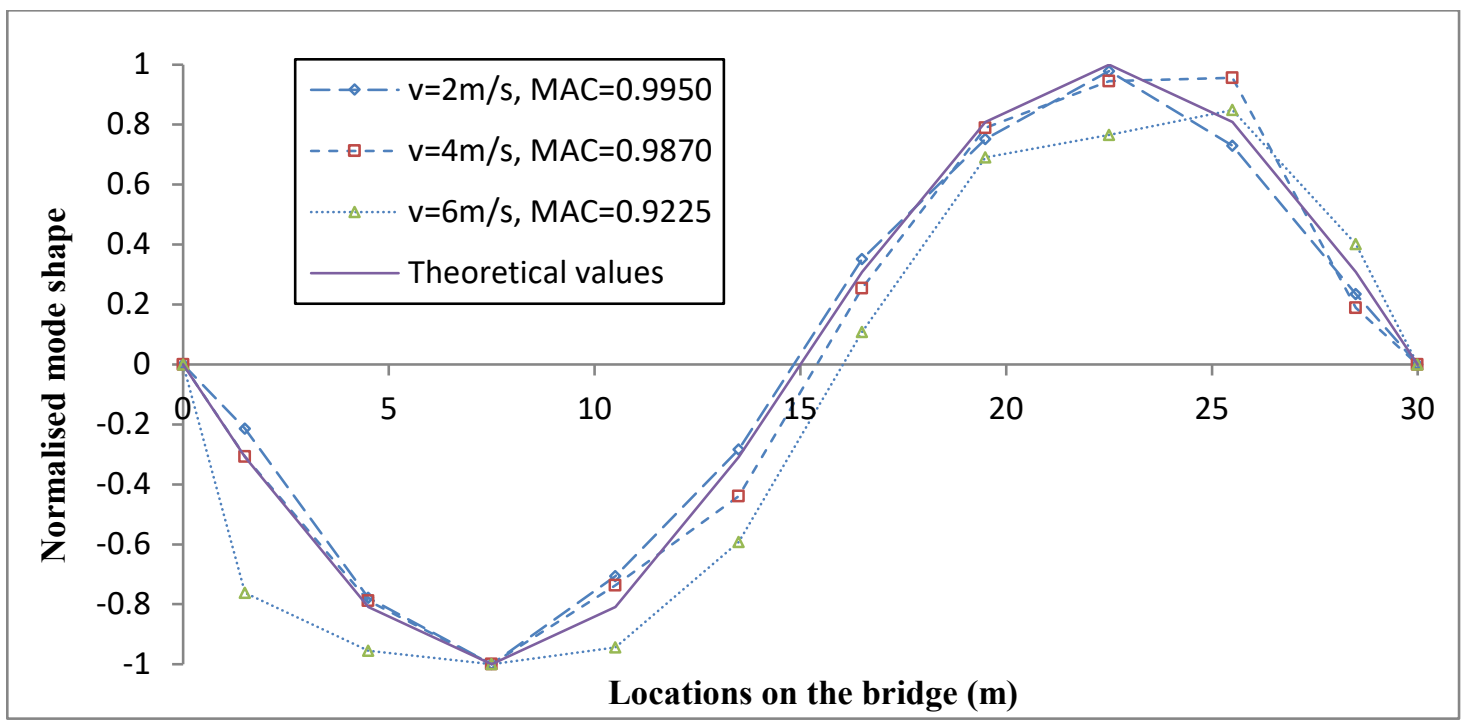

(b) Identified second mode shape

Figure 10 Effect of vehicle speeds on identified results 


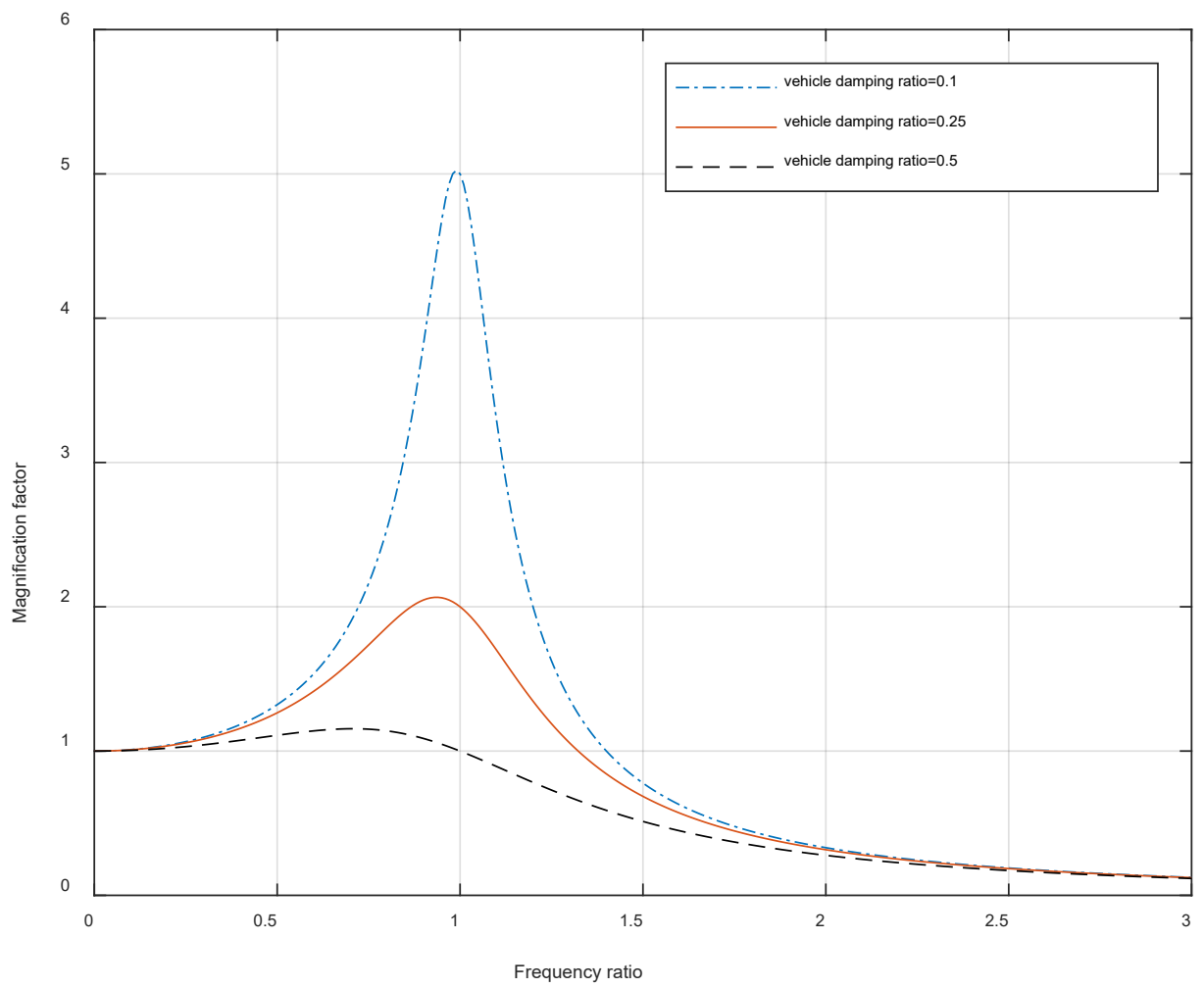

Figure 11 Relation between Magnification Factor with the frequency ratio for different damping ratios

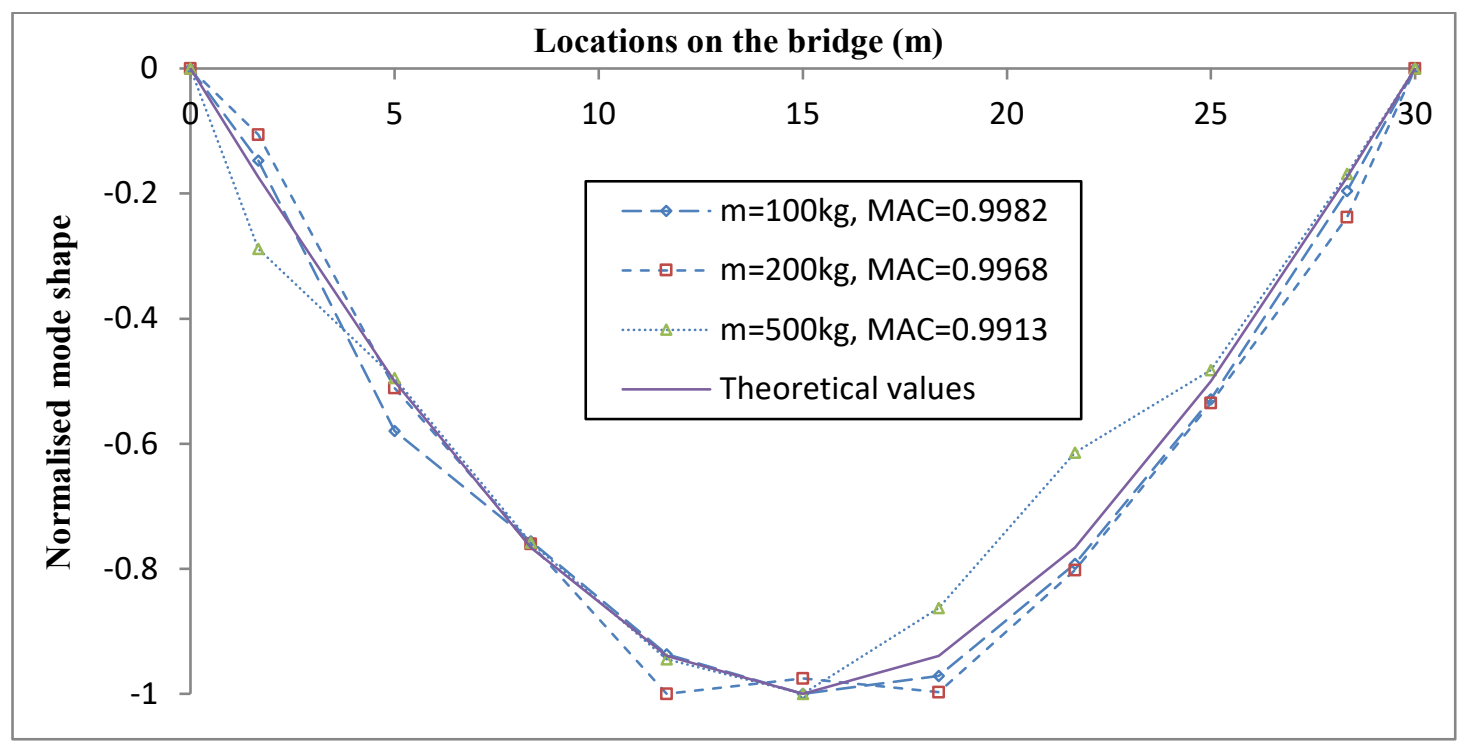

(a) Identified first mode shape 


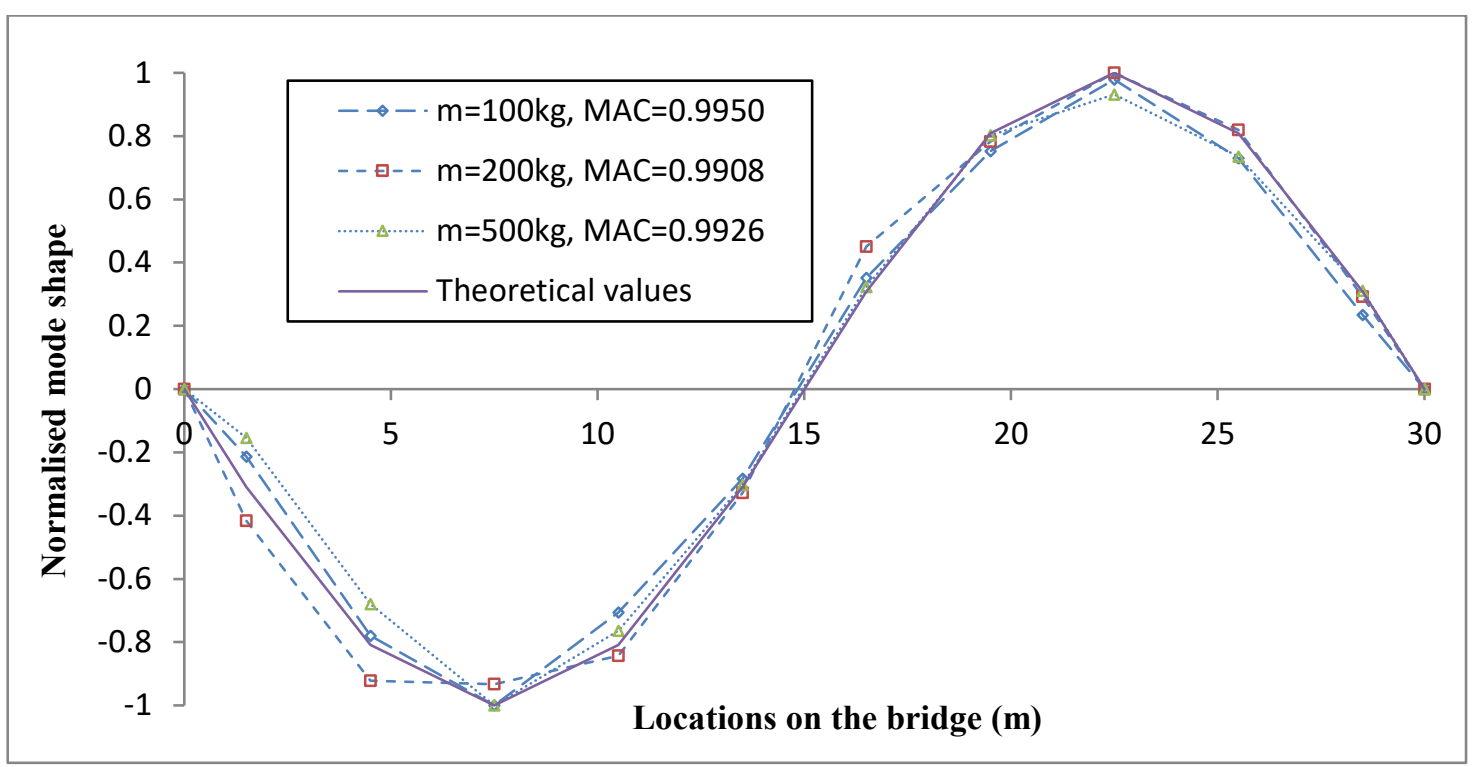

(b) Identified second mode shape

Figure 12 Effect of vehicle weights on identified mode shapes

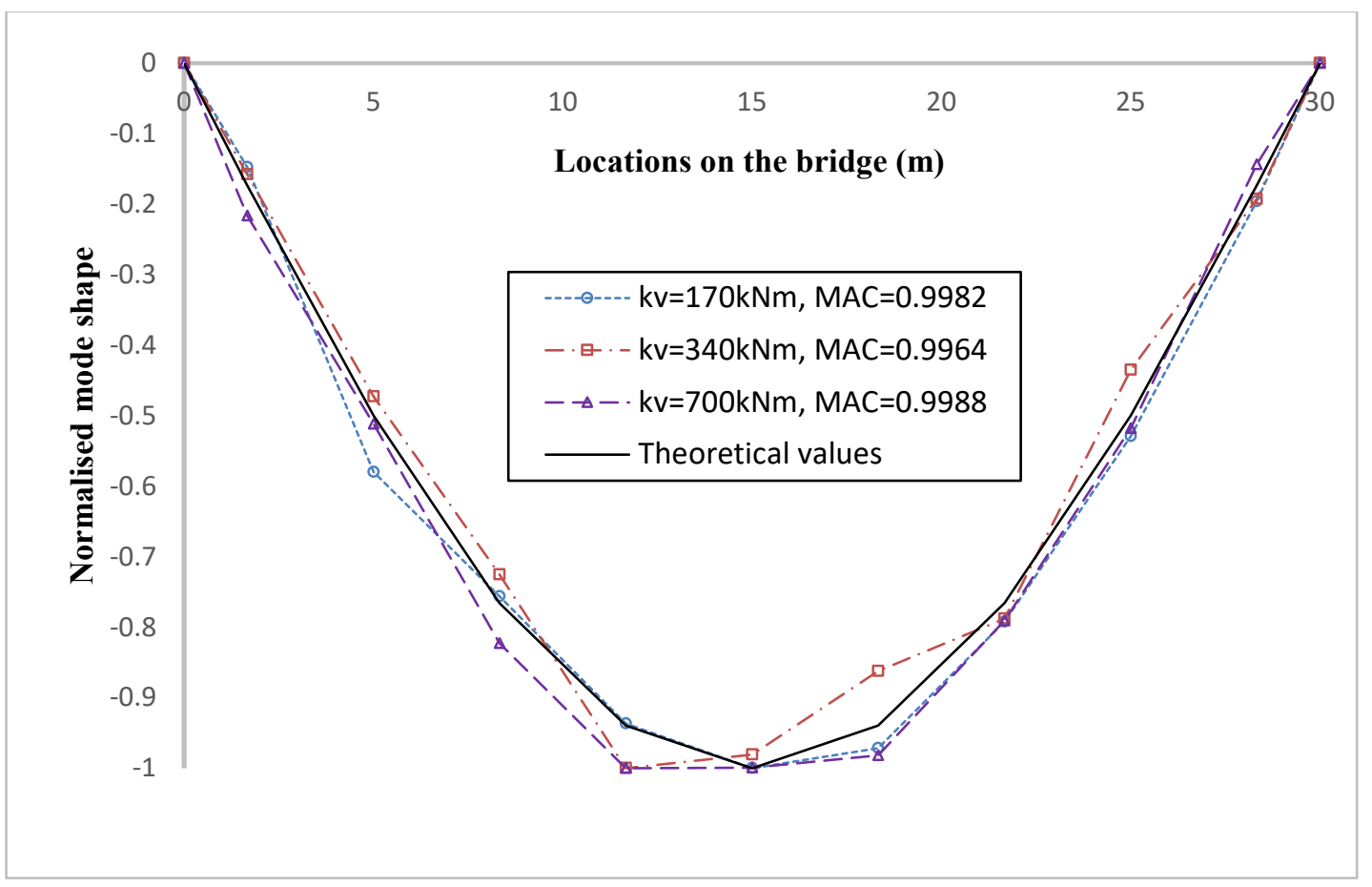

(a) Identified first mode shape 


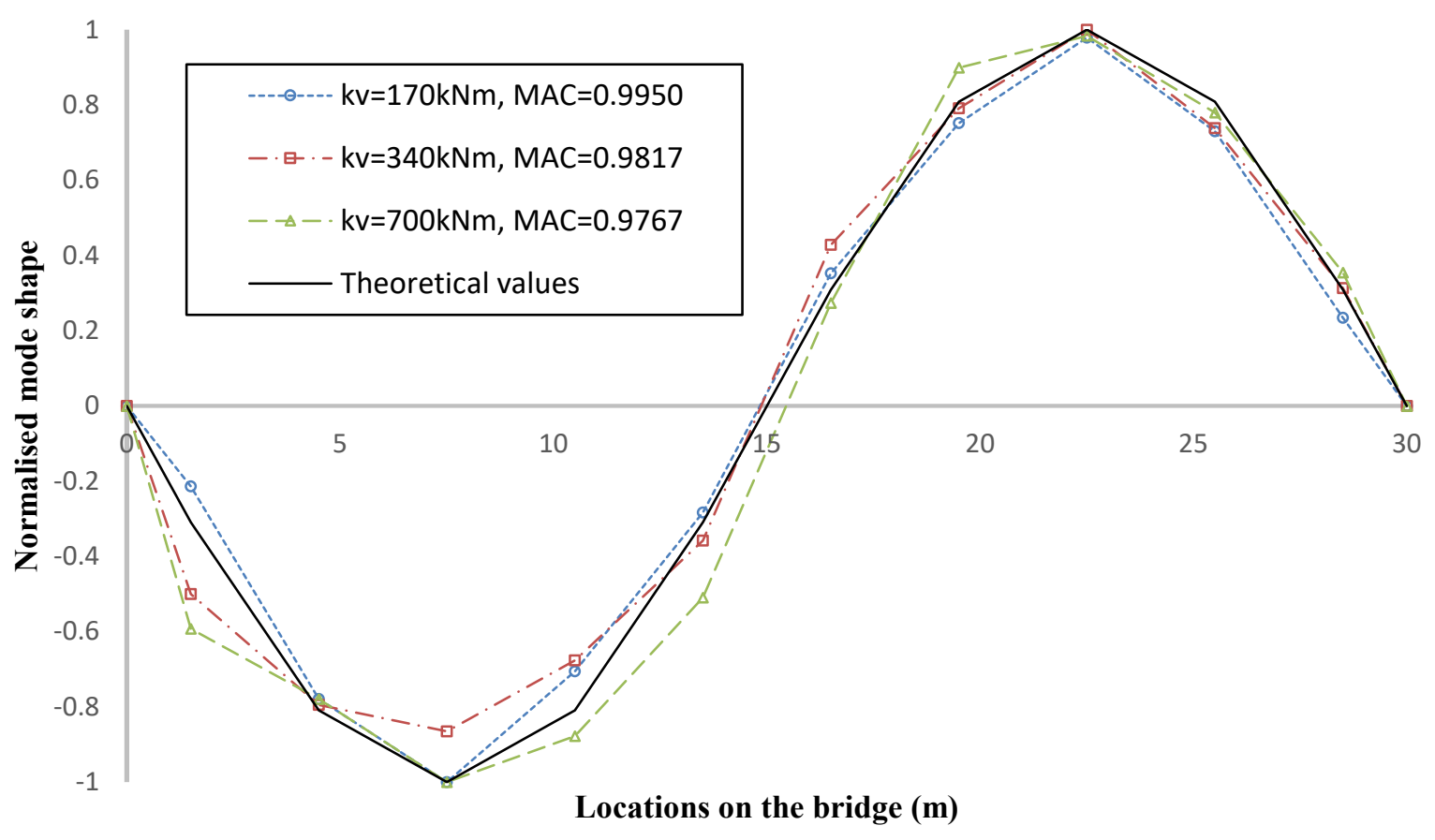

(b) Identified second mode shape

Figure 13 Effect of vehicle stiffness on identified mode shapes

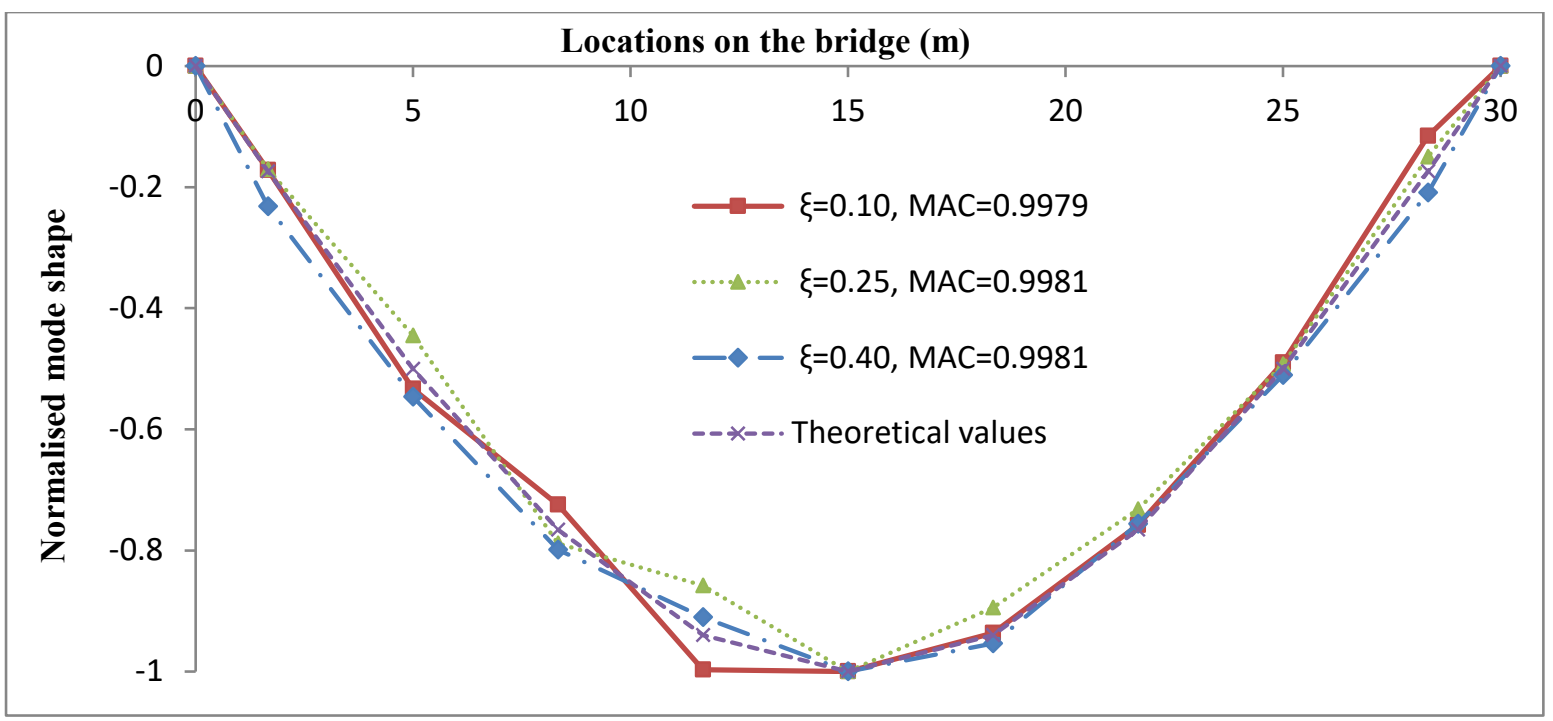

(a) Identified first mode shape 


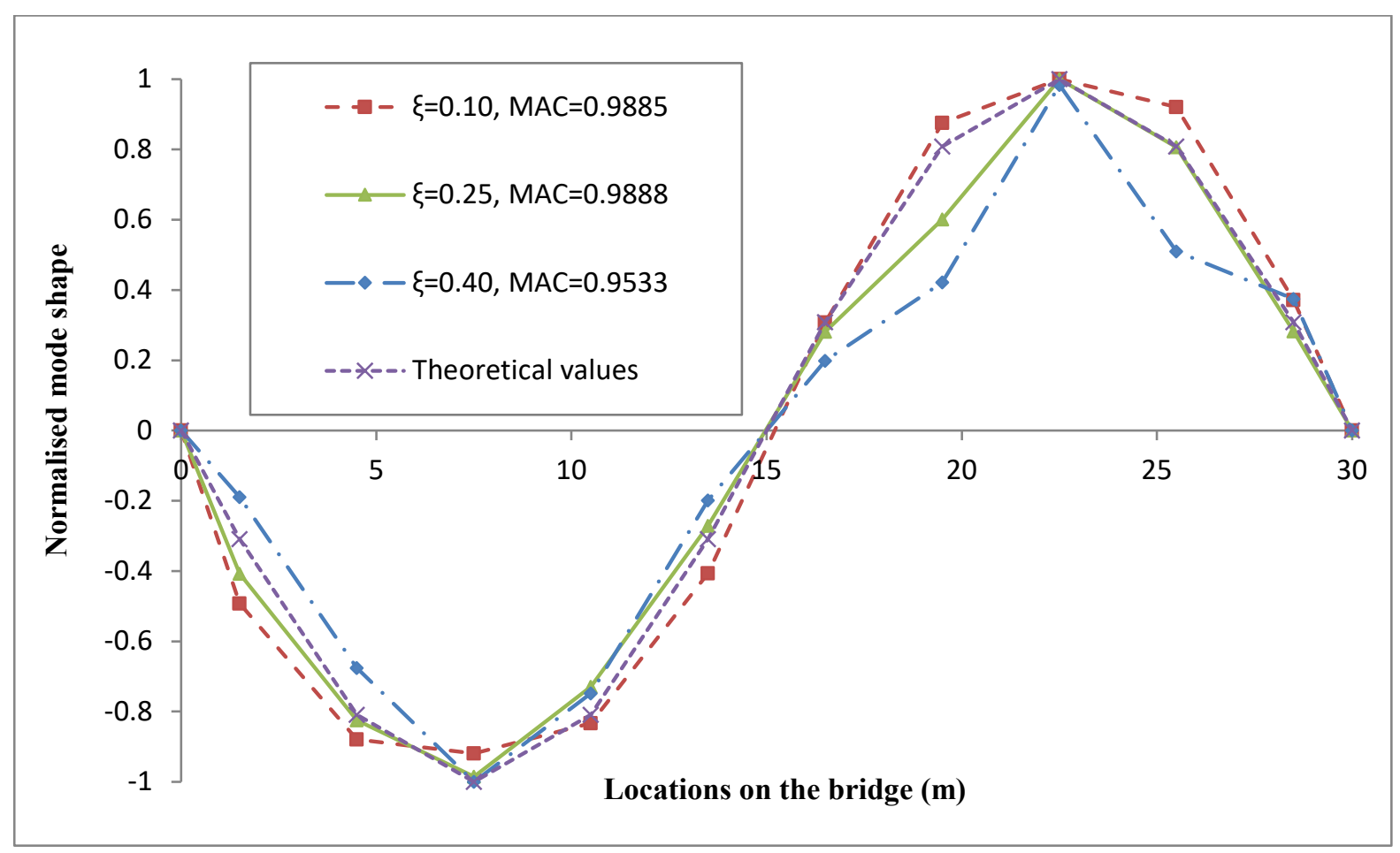

(b) Identified second mode shape

Figure 14 Comparison of identified mode shapes considering different vehicle damping ratios

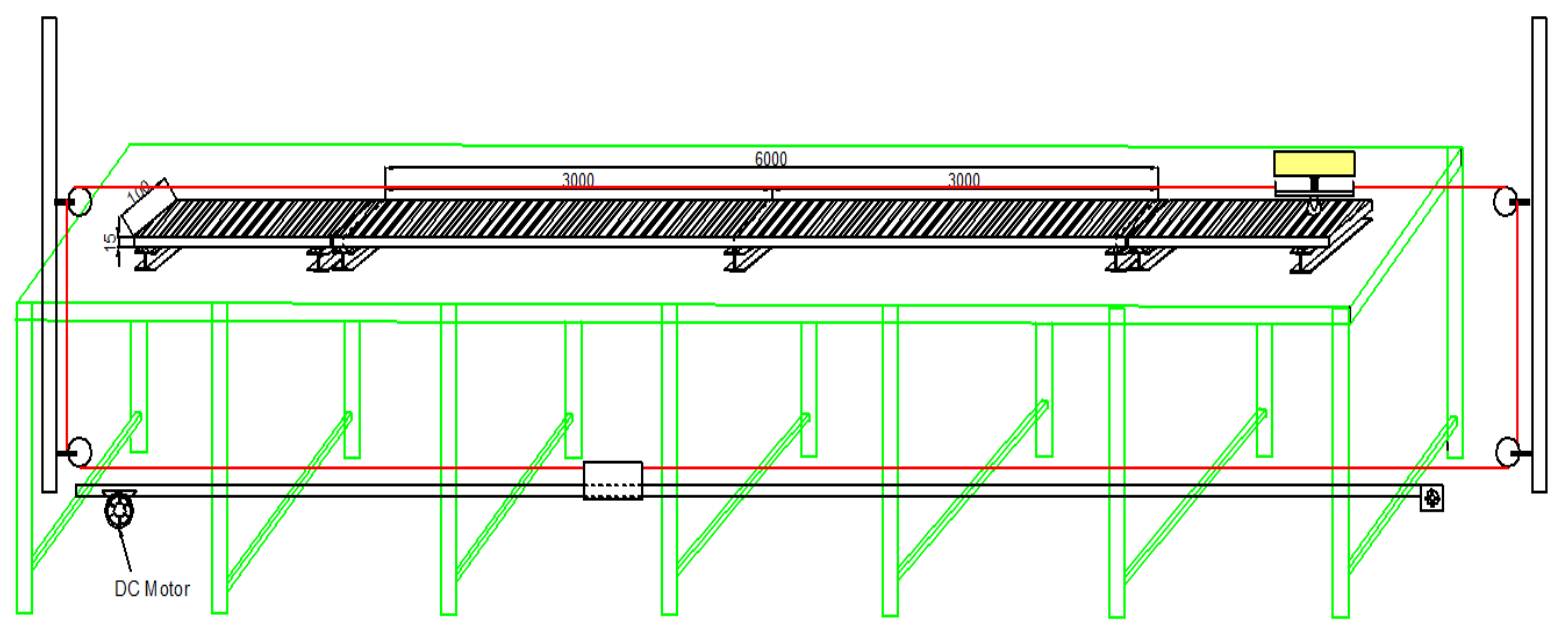

(a) Schematic diagram of the VBI test system in the lab

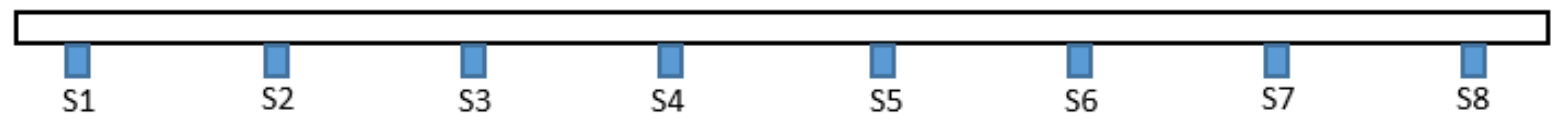

(b) Instrumentation with 8 wired sensors on the beam 


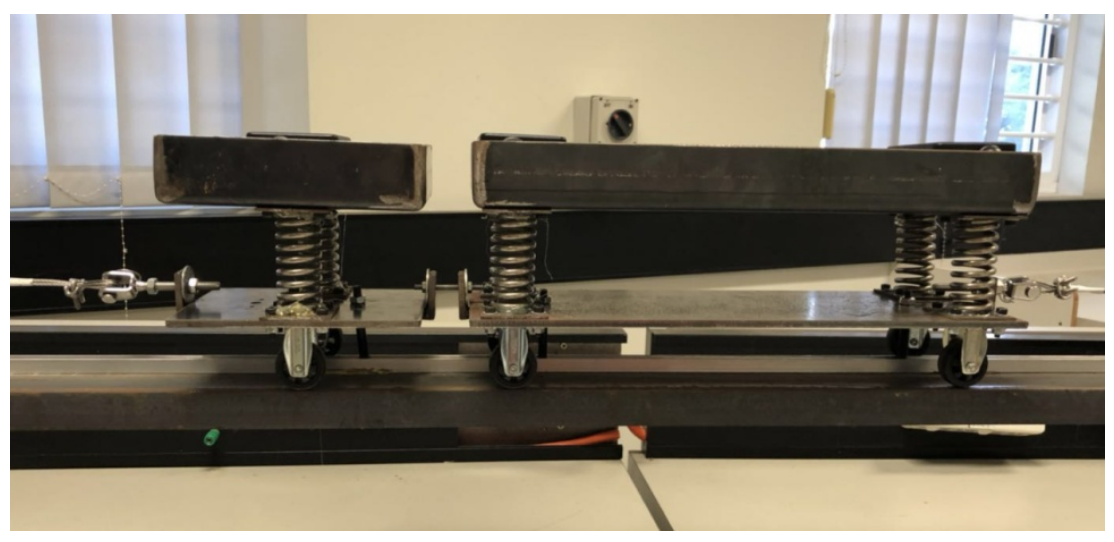

(c) Bridge model subjected to moving vehicles

Figure 15 Vehicle-bridge interaction model

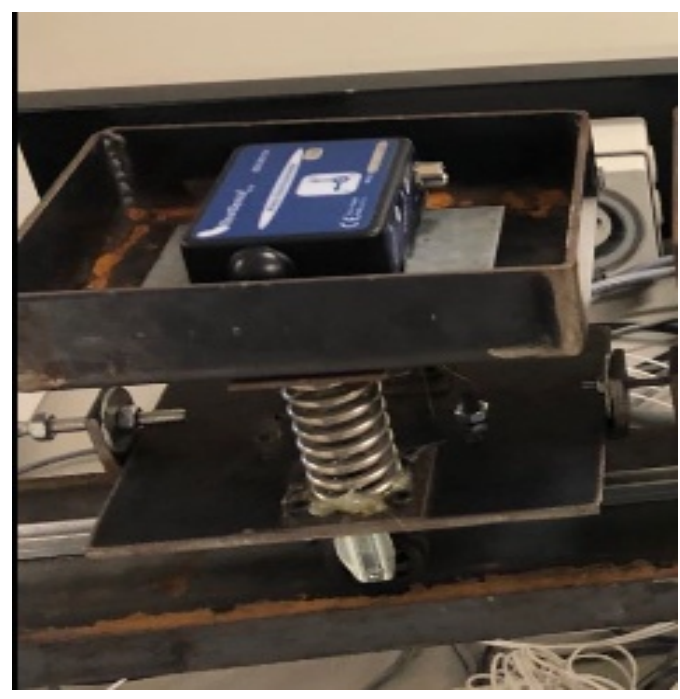

(a) Wireless sensor on the vehicle

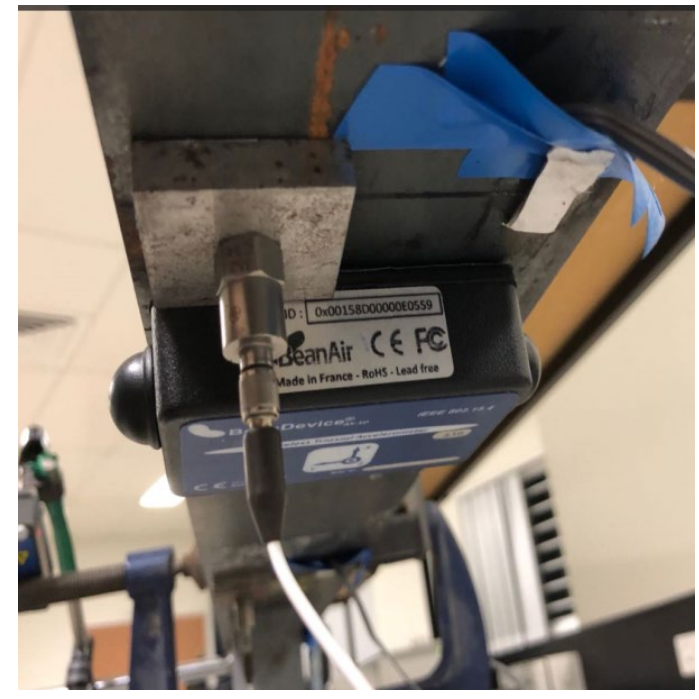

(c) Wireless sensor on the bridge

Figure 16 Installation of the wireless sensors 


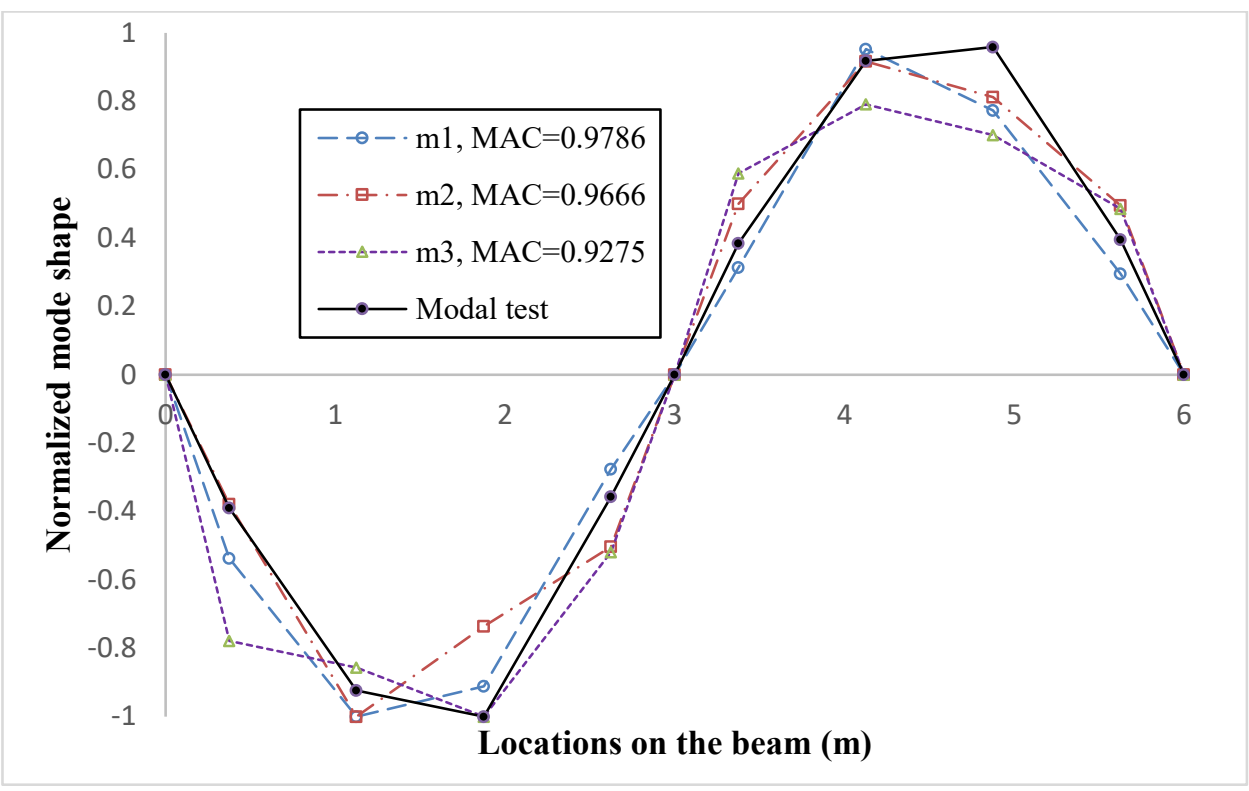

Figure 17 Identified mode shapes using vehicles with different weights

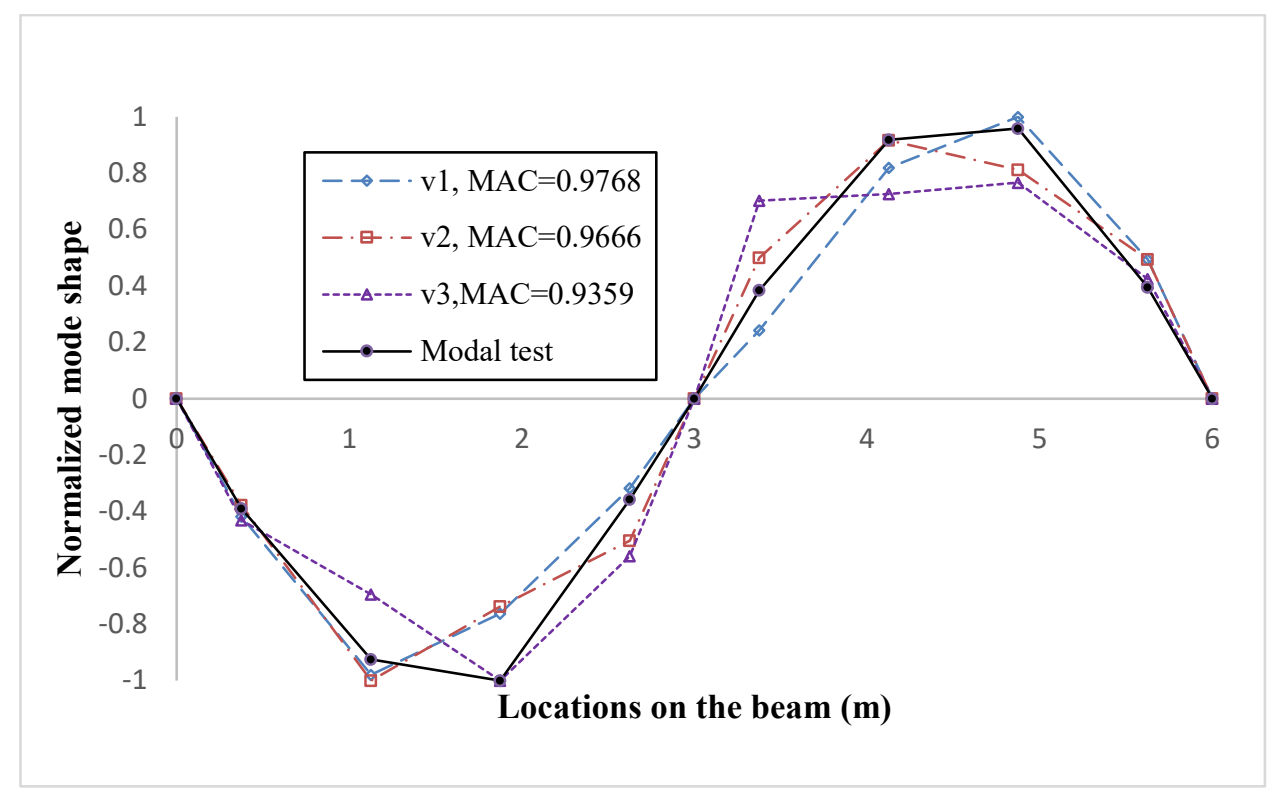

Figure 18 Identified mode shapes with different moving speeds 


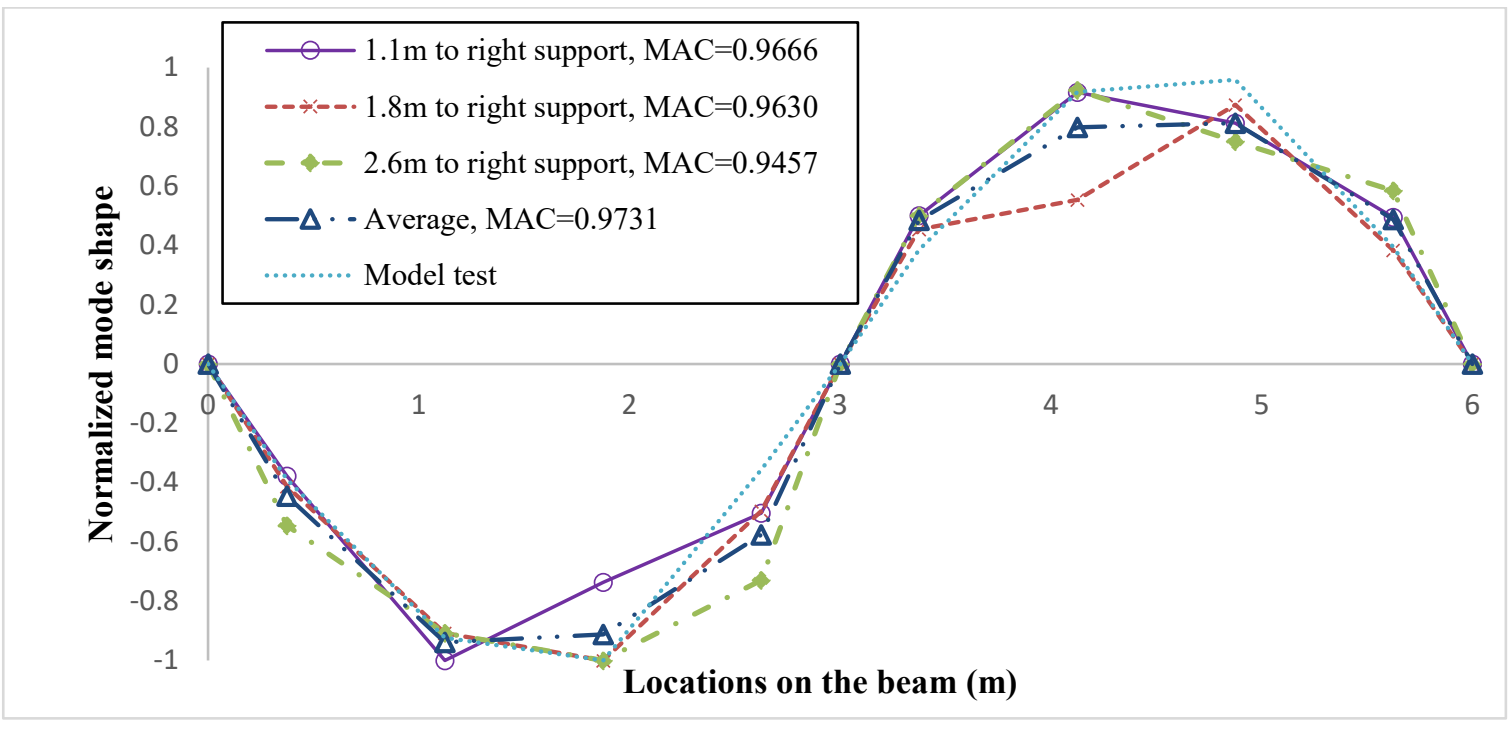

Figure 19 Identified mode shapes considering different locations of reference sensor 